\title{
A Sobolev Poincaré type inequality for integral varifolds
}

\author{
Ulrich Menne
}

Received: 27 November 2008 / Accepted: 28 October 2009 / Published online: 18 November 2009

(C) The Author(s) 2009. This article is published with open access at Springerlink.com

\begin{abstract}
In this work a local inequality is provided which bounds the distance of an integral varifold from a multivalued plane (height) by its tilt and mean curvature. The bounds obtained for the exponents of the Lebesgue spaces involved are shown to be sharp.
\end{abstract}

Mathematics Subject Classification (2000) Primary 49Q15 · Secondary 29B05

\section{Introduction}

Regularity of integral varifolds is often investigated by use of an approximation by Lipschitzian single or multivalued functions. A basic property of such functions is the Sobolev Poincaré inequality. In this paper a similar inequality is established for the varifold itself. An inequality of this type has to involve mean curvature as simple examples demonstrate. Considering a ball centered at a generic point and taking the limit as the radius approaches 0 , the contribution of the mean curvature drops out if and only if the exponents of the Lebesgue spaces involved satisfy a certain inequality. The initial motivation to examine the validity of a Poincaré type inequality was given by a question arising from Schätzle's work in [15], see below.

Basic definitions. First, some definitions will be recalled. Suppose throughout the introduction that $m, n \in \mathbb{N}$ and $U$ is a nonempty, open subset of $\mathbb{R}^{n+m}$. Using [16, Theorem 11.8] as a definition, $\mu$ is a rectifiable [an integral] $n$ varifold in $U$ if and only if $\mu$ is a Radon measure on $U$ and for $\mu$ almost all $x \in U$ there exists an approximate tangent plane $T_{x} \mu \in G(n+m, n)$ with multiplicity $0<\theta^{n}(\mu, x)<\infty$ of $\mu$ at $x$ [and $\theta^{n}(\mu, x) \in \mathbb{N}$ ], $G(n+m, n)$ denoting the set of $n$ dimensional, unoriented planes in $\mathbb{R}^{n+m}$. The distributional first variation of mass of $\mu$ equals 


$$
(\delta \mu)(\eta)=\int \operatorname{div}_{\mu} \eta \mathrm{d} \mu \quad \text { whenever } \eta \in C_{\mathrm{c}}^{1}\left(U, \mathbb{R}^{n+m}\right)
$$

where $\operatorname{div}_{\mu} \eta(x)$ is the trace of $D \eta(x)$ with respect to $T_{x} \mu$. $\|\delta \mu\|$ denotes the total variation measure associated to $\delta \mu$ and $\mu$ is said to be of locally bounded first variation if and only if $\|\delta \mu\|$ is a Radon measure. The tilt-excess and the height-excess of $\mu$ are defined by

$$
\begin{aligned}
\operatorname{tiltex}_{\mu}(x, \varrho, T) & :=\varrho^{-n} \int_{B_{\varrho}(x)}\left|T_{\xi} \mu-T\right|^{2} \mathrm{~d} \mu(\xi), \\
\operatorname{heightex}_{\mu}(x, \varrho, T) & :=\varrho^{-n-2} \int_{B_{\varrho}(x)} \operatorname{dist}(\xi-x, T)^{2} \mathrm{~d} \mu(\xi)
\end{aligned}
$$

whenever $x \in \mathbb{R}^{n+m}, 0<\varrho<\infty, B_{\varrho}(x) \subset U, T \in G(n+m, n)$; here $S \in G(n+m, n)$ is identified with the orthogonal projection of $\mathbb{R}^{n+m}$ onto $S$ and $|\cdot|$ denotes the norm induced by the usual inner product on $\operatorname{Hom}\left(\mathbb{R}^{n+m}, \mathbb{R}^{n+m}\right)$, see e.g. [6, 1.7.9]. From the above definition of a rectifiable $n$ varifold $\mu$ one obtains that $\mu$ almost all of $U$ is covered by a countable collection of $n$ dimensional submanifolds of $\mathbb{R}^{n+m}$ of class $\mathcal{C}^{1}$. This concept is extended to higher orders of differentiability by adapting a definition of Anzellotti and Serapioni in [3] as follows: A rectifiable $n$ varifold $\mu$ in $U$ is called countably rectifiable of class $\mathcal{C}^{k, \alpha}$ $\left[\mathcal{C}^{k}\right], k \in \mathbb{N}, 0<\alpha \leq 1$, if and only if there exists a countable collection of $n$ dimensional submanifolds of $\mathbb{R}^{n+m}$ of class $\mathcal{C}^{k, \alpha}\left[\mathcal{C}^{k}\right]$ covering $\mu$ almost all of $U$. Throughout the introduction this will be abbreviated to $\mathcal{C}^{k, \alpha}\left[\mathcal{C}^{k}\right]$ rectifiability. Note that $\mathcal{C}^{k, 1}$ rectifiability and $\mathcal{C}^{k+1}$ rectifiability agree by $[6,3.1 .15]$.

Known results. Decays of tilt-excess or height-excess have been successfully used by Allard [1], Brakke [4] and Schätzle [14,15]. The link to $\mathcal{C}^{2}$ rectifiability is provided by Schätzle [15]. In order to explain some of these results, a mean curvature condition is introduced. An integral $n$ varifold in $U$ is said to satisfy $\left(H_{p}\right), 1 \leq p \leq \infty$, if and only if either $p>1$ and for some $\vec{H}_{\mu} \in L_{\mathrm{loc}}^{p}\left(\mu, \mathbb{R}^{n+m}\right)$, called the generalised mean curvature of $\mu$,

$$
(\delta \mu)(\eta)=-\int \vec{H}_{\mu} \bullet \eta \mathrm{d} \mu \quad \text { whenever } \eta \in C_{\mathrm{c}}^{1}\left(U, \mathbb{R}^{n+m}\right)
$$

or $p=1$ and

$$
\mu \text { is of locally bounded first variation; }
$$

here $\bullet$ denotes the usual inner product on $\mathbb{R}^{n+m}$. Brakke has shown in $[4,5.7]$ that

$$
\operatorname{tiltex}_{\mu}\left(x, \varrho, T_{x} \mu\right)=o_{x}(\varrho), \text { heightex }_{\mu}\left(x, \varrho, T_{x} \mu\right)=o_{x}(\varrho) \text { as } \varrho \downarrow 0
$$

for $\mu$ almost every $x \in U$ provided $\mu$ satisfies $\left(H_{1}\right)$ and

$$
\operatorname{tiltex}_{\mu}\left(x, \varrho, T_{x} \mu\right)=o_{x}\left(\varrho^{2-\varepsilon}\right) \text {, heightex }{ }_{\mu}\left(x, \varrho, T_{x} \mu\right)=o_{x}\left(\varrho^{2-\varepsilon}\right) \text { as } \varrho \downarrow 0
$$

for every $\varepsilon>0$ for $\mu$ almost every $x \in U$ provided $\mu$ satisfies $\left(H_{2}\right)$. In case of codimension 1 and $p>n$ Schätzle has proved the following result yielding optimal decay rates.

Theorem 5.1 in [14] If $m=1, p>n, p \geq 2$, and $\mu$ is an integral $n$ varifold in $U$ satisfying $\left(H_{p}\right)$, then

$$
\operatorname{tiltex}_{\mu}\left(x, \varrho, T_{x} \mu\right)=O_{x}\left(\varrho^{2}\right), \operatorname{heightex}_{\mu}\left(x, \varrho, T_{x} \mu\right)=O_{x}\left(\varrho^{2}\right) \text { as } \varrho \downarrow 0
$$

for $\mu$ almost all $x \in U$. 
The importance of the improvement from $2-\varepsilon$ to 2 stems mainly from the fact that the quadratic decay of tilt-excess can be used to compute the mean curvature vector $\vec{H}_{\mu}$ in terms of the local geometry of $\mu$ which had already been observed by Schätzle in [13, Lemma 6.3]. In [15] Schätzle provides the above mentioned link to $\mathcal{C}^{2}$ rectifiability as follows:

Theorem 3.1 in [15] If $\mu$ is an integral $n$ varifold in $U$ satisfying $\left(H_{2}\right)$ then the following two statements are equivalent:

(1) $\mu$ is $\mathcal{C}^{2}$ rectifiable.

(2) For $\mu$ almost every $x \in U$ there holds

$$
\operatorname{tiltex}_{\mu}\left(x, \varrho, T_{x} \mu\right)=O_{x}\left(\varrho^{2}\right) \text {, heightex } \mu\left(x, \varrho, T_{x} \mu\right)=O_{x}\left(\varrho^{2}\right) \text { as } \varrho \downarrow 0 .
$$

The quadratic decay of heightex ${ }_{\mu}$ implies $\mathcal{C}^{2}$ rectifiability without the condition $\left(H_{2}\right)$ as was noted in [15]. However, (1) would not imply (2) if $\mu$ were merely required to satisfy $\left(H_{p}\right)$ for some $p$ with $1 \leq p<2 n /(n+2)$, an example was be provided in [11, 1.5]. On the other hand, it is evident from the Caccioppoli type inequality relating tiltex ${ }_{\mu}$ to heightex ${ }_{\mu}$ and mean curvature, see e.g. Brakke $[4,5.5]$, that quadratic decay of heightex ${ }_{\mu}$ implies quadratic decay for tiltex ${ }_{\mu}$ under the condition $\left(\mathrm{H}_{2}\right)$. This leads to the following question:

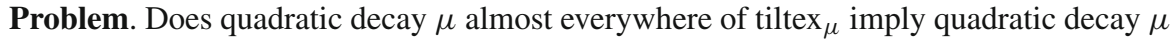
almost everywhere of heightex ${ }_{\mu}$ under the condition $\left(H_{2}\right)$ ? More generally, suppose that $\mu$ is an integral $n$ varifold in $U$ satisfying $\left(H_{p}\right), 1 \leq p \leq \infty$, and $0<\alpha \leq 1,1 \leq q<\infty$. Does

$$
\limsup _{r \downarrow 0} r^{-\alpha-n / q}\left(\int_{B_{r}(x)}\left|T_{\xi} \mu-T_{x} \mu\right|^{q} \mathrm{~d} \mu(\xi)\right)^{1 / q}<\infty
$$

for $\mu$ almost all $x \in U$ imply

$$
\limsup _{r \downarrow 0} r^{-1-\alpha-n / q}\left(\int_{B_{r}(x)} \operatorname{dist}\left(\xi-x, T_{x} \mu\right)^{q} \mathrm{~d} \mu(\xi)\right)^{1 / q}<\infty
$$

for $\mu$ almost all $x \in U$ ?

Results of the present paper. The answer to the second question will be shown in 4.114.13 to be in the affirmative if and only if either $p \geq n$ or $p<n$ and $\alpha q \leq n p /(n-p)$, yielding in particular a positive answer to the first question. The main task is to prove the following theorem which in fact provides a quantitative estimate together with the usual embedding in $L^{q}$ spaces.

Theorem 4.11 Suppose $Q \in \mathbb{N}, 0<\alpha \leq 1,1 \leq p \leq n$, and $\mu$ is an integral $n$ varifold in $U$ satisfying $\left(H_{p}\right)$.

Then the following two statements hold:

(1) If $p<n, 1 \leq q_{1}<n, 1 \leq q_{2} \leq \min \left\{\frac{n q_{1}}{n-q_{1}}, \frac{1}{\alpha} \cdot \frac{n p}{n-p}\right\}$, then for $\mu$ almost all $a \in U$ with $\theta^{n}(\mu, a)=Q$ there holds

$$
\begin{aligned}
& \limsup _{r \downarrow 0} r^{-\alpha-1-n / q_{2}}\left\|\operatorname{dist}\left(\cdot-a, T_{a} \mu\right)\right\|_{L^{q_{2}}\left(\mu\left\llcorner B_{r}(a)\right)\right.} \\
& \leq \Gamma_{(1)} \limsup _{r \downarrow 0} r^{-\alpha-n / q_{1}}\left\|T_{\mu}-T_{a} \mu\right\|_{L^{q_{1}}\left(\mu\left\llcorner B_{r}(a)\right)\right.}
\end{aligned}
$$

where $\Gamma_{(1)}$ is a positive, finite number depending only on $m, n, Q, q_{1}$, and $q_{2}$. 
(2) If $p=n, n<q \leq \infty$, then for $\mu$ almost all $a \in U$ with $\theta^{n}(\mu, a)=Q$ there holds

$$
\begin{aligned}
& \limsup _{r \downarrow 0} r^{-\alpha-1}\left\|\operatorname{dist}\left(\cdot-a, T_{a} \mu\right)\right\|_{L^{\infty}\left(\mu\left\llcorner B_{r}(a)\right)\right.} \\
& \leq \Gamma_{(2)} \limsup _{r \downarrow 0} r^{-\alpha-n / q}\left\|T_{\mu}-T_{a} \mu\right\|_{L^{q}\left(\mu\left\llcorner B_{r}(a)\right)\right.}
\end{aligned}
$$

where $\Gamma_{(2)}$ is a positive, finite number depending only on $m, n, Q$, and $q$.

Here $T_{\mu}$ denotes the function mapping $x$ to $T_{x} \mu$ whenever the latter exists. The connection to higher order rectifiability is provided by the following simple adaption of Schätzle [15, Appendix A] by use of [17, VI.2.2.2, VI.2.3.1-3].

Lemma Suppose $0<\alpha \leq 1, \mu$ is a rectifiable $n$ varifold in $U$, and $A$ denotes the set of all $x \in U$ such that $T_{x} \mu$ exists and

$$
\underset{\varrho \downarrow 0}{\limsup } \varrho^{-n-1-\alpha} \int_{B_{\varrho}(x)} \operatorname{dist}\left(\xi-x, T_{x} \mu\right) \mathrm{d} \mu(\xi)<\infty .
$$

Then $\mu\left\llcorner A\right.$ is $\mathcal{C}^{1, \alpha}$ rectifiable.

The analog of Theorem 4.11 in the case of weakly differentiable functions can be proved simply by using the Sobolev Poincaré inequality in conjunction with an iteration procedure. In the present case, however, the curvature condition is needed to exclude a behaviour like the one shown by the function $f: \mathbb{R} \rightarrow \mathbb{R}$ defined by

$$
f(x)=\sum_{i=0}^{\infty}\left(2^{-i}\right) \chi_{\left[2^{-i-1}, 2^{-i}[\right.}(x) \quad \text { whenever } x \in \mathbb{R}
$$

at 0 ; in fact an example of this behaviour occurring on a set of positive $\mathcal{L}^{1}$ measure is provided by $f^{1 / 2} \circ g$ where $g$ is the distance function from a compact set $C$ such that $\mathcal{L}^{1}(C)>0$ and for some $0<\lambda<1$

$$
\liminf _{r \downarrow 0} r^{-3 / 2} \mathcal{L}^{1}([x+\lambda r, x+r[\sim C)>0 \quad \text { whenever } x \in C .
$$

Therefore the strategy to prove Theorem 4.11 is to provide a special Sobolev Poincaré type inequality for integral varifolds involving curvature, see Theorem 4.4. In the construction weakly differentiable functions are replaced by Lipschitzian $Q$ valued functions, a $Q$ valued function being a function with values in $Q_{Q}\left(\mathbb{R}^{m}\right) \cong\left(\mathbb{R}^{m}\right)^{Q} / \sim$ where $\sim$ is induced by the action of the group of permutations of $\{1, \ldots, Q\}$ on $\left(\mathbb{R}^{m}\right)^{Q}$.

Method of proof. Roughly speaking, the construction performed in a ball $B_{r}(a) \subset U$ proceeds as follows. Firstly, a graphical part $G$ of $\mu$ in $B_{r}(a)$ is singled out. The complement of $G$ can be controlled in mass by the curvature, whereas its geometry cannot be controlled in a suitable way as may be seen from the example in $[11,1.2]$ used to demonstrate the sharpness of the curvature condition. On the graphical part $G$ the varifold $\mu$ might not quite correspond to the graph of a $Q$ valued function but still have "holes" or "missing layers". Nevertheless, it will be shown that, on $G, \mu$ behaves just enough like a $Q$ valued function to make it possible to reduce the problem to this case. Finally, for $Q$ valued functions Almgren's bi Lipschitzian equivalence of $Q_{Q}\left(\mathbb{R}^{m}\right)$ to a subset of $\mathbb{R}^{P Q}$ for some $P \in \mathbb{N}$ which is a Lipschitz retract of the whole space directly yields a Sobolev Poincaré inequality. More details about the technical difficulties occurring in the construction and how they are solved will be given at the beginning of Sect. 3 . 
Organisation of the paper. In Sect. 2 some basic properties of $Q$ valued functions are provided. In Sect. 3 the approximation of $\mu$ by a $Q$ valued function is constructed. In Sect. 4 the approximation is used to prove the Theorems 4.4 and 4.11 .

The results have been previously published in the author's $\mathrm{PhD}$ thesis, see [10].

Additional notation. The notation follows [16] and, concerning $Q$ valued functions, Almgren [2, T.1(20), 1.1(1), (9)-(11)]. In particular, the functions $\eta_{a, r}: \mathbb{R}^{n+m} \rightarrow \mathbb{R}^{n+m}$ are given by $\eta_{a, r}(x)=r^{-1}(x-a)$ for $a, x \in \mathbb{R}^{n+m}, 0<r<\infty$ and $N(k)$ denotes the best constant in Besicovitch's covering theorem in $\mathbb{R}^{k}$, see [16, Lemma 4.6]. Additionally to the symbols already defined, $\operatorname{im} f, \operatorname{dmn} f$ and $f \mid A$ denote the image of $f$, the domain of $f$ and the restriction of $f$ to a set $A$ whenever $f$ is a function, $T^{\perp}$ is the orthogonal complement of $T$ for $T \in G(n+m, n), \gamma_{n}$ denotes the best constant in the isoperimetric inequality as defined in Definition 3.3, and $f(\phi)$ denotes the ordinary push forward of a measure $\phi$ by a function $f$, i.e. $f(\phi)(A):=\phi\left(f^{-1}(A)\right)$ whenever $A \subset Y$, if $\phi$ is a measure on $X$ and $f: X \rightarrow Y$. Definitions are denoted by ' $=$ ' or, if clarity makes it desirable, by $':=$ '. To simplify verification, in case a statement asserts the existence of a constant, small $(\varepsilon)$ or large $(\Gamma)$, depending on certain parameters this number will be referred to by using the number of the statement as index and what is supposed to replace the parameters in the order of their appearance given in parentheses, for example $\varepsilon_{3.4}\left(m, n, 1-\delta_{3} / 2\right)$. Finally, as in Almgren [2, T.1 (23)] the join $f \bowtie g$ of two maps $f: A \rightarrow B$ and $g: A \rightarrow C$ is defined by $(f \bowtie g)(a)=(f(a), g(a))$ for $a \in A$. For the convenience of the reader the notation used from Almgren [2] is recalled in 2.1 and 2.2.

\section{Basic facts for $Q_{Q}\left(\mathbb{R}^{m}\right)$ valued functions}

The purpose of this section is to collect some results concerning $Q$ valued functions (cf. Almgren [2]). Among them an elementary but useful decomposition of a Lipschitzian $Q$ valued function into a countable collection of ordinary Lipschitzian functions is proved in Theorem 2.5. This decomposition directly entails both the rectifiability of the $Q$ valued graph which had been proved by Almgren using the compactness theorem for integral currents and also a simple proof of Stepanoff's theorem for $Q$ valued functions in Theorem 2.8. Other proofs of the special case of Rademacher's theorem avoiding Almgren's bi Lipschitzian embedding of $Q_{Q}\left(\mathbb{R}^{n+m}\right)$ into a Euclidean space can be found in Goblet [8] and De Lellis and Spadaro [5, Theorem 1.13]. Also, the Sobolev Poincaré inequality for $Q$ valued functions and a Lipschitz extension theorem is given, see Theorems 2.10 and 2.12 .

2.1 (cf. [2, T.1(20), 1.1(1)]) Suppose $Q \in \mathbb{N}$ and $V$ is a finite dimensional Euclidean vector space.

For $x \in V$ define $\llbracket x \rrbracket$ as the 0 dimensional current in $V$ associated to the Dirac measure at $x$, i.e. $\llbracket x \rrbracket(f)=f(x)$ for $f \in C_{c}^{\infty}(V)$. The space $Q_{Q}(V)$ is defined to be the set of all 0 dimensional integral currents $R$ such that $R=\sum_{i=1}^{Q} \llbracket x_{i} \rrbracket$ for some $x_{1}, \ldots, x_{Q} \in V$. A metric $\mathcal{G}$ on $Q_{Q}(V)$ is defined such that

$$
\mathcal{G}\left(\sum_{i=1}^{Q} \llbracket x_{i} \rrbracket, \sum_{i=1}^{Q} \llbracket y_{i} \rrbracket\right)=\min \left\{\left(\sum_{i=1}^{Q}\left|x_{i}-y_{\pi(i)}\right|^{2}\right)^{1 / 2}: \pi \in \mathcal{S}(Q)\right\}
$$

whenever $x_{1}, \ldots, x_{Q}, y_{1}, \ldots, y_{Q} \in V$ where $\mathcal{S}(Q)$ denotes the set of permutations of $\{1, \ldots, Q\}$. Note that in case $T=\sum_{i=1}^{Q} \llbracket x_{i} \rrbracket$ for some $x_{1}, \ldots, x_{Q} \in V$, 


$$
\begin{aligned}
\operatorname{spt} T & =\left\{x_{1}, \ldots, x_{Q}\right\}, \quad\|T\|=\sum_{i=1}^{Q} \delta_{x_{i}}, \\
\theta^{0}(\|T\|, x) & =\#\left\{i: x_{i}=x\right\} \quad \text { whenever } x \in V
\end{aligned}
$$

where $\delta_{x}$ denotes the Dirac measure at $x$.

Additionally to Almgren's notation, define the $Q$ valued graph of $f$ by

$$
\operatorname{graph}_{Q} f=\{(x, v) \in X \times V: v \in \operatorname{spt} f(x)\} .
$$

whenever $f: X \rightarrow Q_{Q}(V)$.

2.2 (cf. [2, 1.1(9)-(11)]) Suppose $m, n, Q \in \mathbb{N}$.

A function $f: \mathbb{R}^{n} \rightarrow Q_{Q}\left(\mathbb{R}^{m}\right)$ is called affine if and only if there exist affine functions $f_{i}: \mathbb{R}^{n} \rightarrow \mathbb{R}^{m}, i=1, \ldots, Q$ such that

$$
f(x)=\sum_{i=1}^{Q} \llbracket f_{i}(x) \rrbracket \quad \text { whenever } x \in \mathbb{R}^{n} .
$$

$f_{1}, \ldots, f_{Q}$ are uniquely determined up to order. One defines semi norms such that

$$
|f|=\left(\sum_{i=1}^{Q}\left|D f_{i}(a)\right|^{2}\right)^{1 / 2}, \quad\|f\|=\limsup _{x \rightarrow a} \mathcal{G}(f(x), f(a)) /|x-a|
$$

whenever $a \in \mathbb{R}^{n}$. Among their basic properties are the following inequalities:

$$
\begin{aligned}
\max \left\{\left\|D f_{i}(a)\right\|: i=1, \ldots, Q\right\} & \leq\|f\| \leq Q^{1 / 2} \max \left\{\left\|D f_{i}(a)\right\|: i=1, \ldots, Q\right\}, \\
|f| & \leq m^{1 / 2} Q^{1 / 2}\|f\|, \quad \operatorname{Lip} f=\|f\| \leq|f| .
\end{aligned}
$$

Let $a \in A \subset \mathbb{R}^{n}, f: A \rightarrow Q_{Q}\left(\mathbb{R}^{m}\right)$. $f$ is called affinely approximable at $a$ if and only if $A$ contains a neighbourhood of $a$ and there exists an affine function $g: \mathbb{R}^{n} \rightarrow Q_{Q}\left(\mathbb{R}^{m}\right)$ such that

$$
\lim _{x \rightarrow a} \mathcal{G}(f(x), g(x)) /|x-a|=0 .
$$

$f$ is called approximately affinely approximable at $a$ if and only if there exists an affine function $g: \mathbb{R}^{n} \rightarrow Q_{Q}\left(\mathbb{R}^{m}\right)$ such that (see $[6,2.9 .12,3.1 .2]$ )

$$
\text { ap } \lim _{x \rightarrow a} \mathcal{G}(f(x), g(x)) /|x-a|=0 .
$$

The function $g$ is unique in both cases and denoted by $A f(a)$ and ap $A f(a)$ respectively. $f$ is called strongly affinely approximable at $a$ if and only if $A f(a)$ has the following property: If $A f(a)(x)=\sum_{i=1}^{Q} \llbracket g_{i}(x) \rrbracket$ whenever $x \in \mathbb{R}^{n}$ for some affine functions $g_{i}: \mathbb{R}^{n} \rightarrow \mathbb{R}^{m}$ and $g_{i}(a)=g_{j}(a)$ for some $i$ and $j$, then $D g_{i}(a)=D g_{j}(a)$. Similarly, one defines approximately strongly affinely approximable at $a$.

If $f$ is affinely approximable [approximately affinely approximable] at $a$, then

$$
\|A f(a)\| \leq \operatorname{Lip} f \quad[\| \text { ap } A f(a) \| \leq \operatorname{Lip} f] .
$$

Definition 2.3 (cf. [6, 3.3.1]) For $m, n, Q \in \mathbb{N}, a \in \mathbb{R}^{n+m}, 0 \leq r<\infty, V \in G(n+m, m)$, and $0<s<1$ let

$$
X(a, r, V, s):=\left\{x \in \mathbb{R}^{n+m}: s^{-1} \operatorname{dist}(x-a, V)<|x-a|<r\right\} .
$$


2.4 Sometimes it will be made of use the fact that $f \subset \operatorname{dmn} f \times \operatorname{im} f$ for any function $f$ in order to simplify notation.

Theorem 2.5 Suppose $m, n, Q \in \mathbb{N}, A$ is $\mathcal{L}^{n}$ measurable, and $f: A \rightarrow Q_{Q}\left(\mathbb{R}^{m}\right)$ is a Lipschitzian function.

Then the following two conclusions hold:

(1) There exists a countable set I and for each $i \in I$ a function $f_{i}: A_{i} \rightarrow \mathbb{R}^{m}$ such that $A_{i}$ is $\mathcal{L}^{n}$ measurable, $A_{i} \subset A$, Lip $f_{i} \leq \operatorname{Lip} f$ and

$$
\#\left\{i: f_{i}(x)=y\right\}=\theta^{0}(\|f(x)\|, y) \text { whenever }(x, y) \in A \times \mathbb{R}^{m} .
$$

If $A$ is a Borel set, then $f_{i}$ and $A_{i}$ may be chosen to be Borel sets in $\mathbb{R}^{n} \times \mathbb{R}^{m}$ and $\mathbb{R}^{n}$ respectively, see 2.4 .

(2) The function $f$ is approximately strongly affinely approximable and whenever I and $f_{i}$ satisfy the conditions of (1)

$$
\text { ap } A f(a)(v)=\sum_{i \in I(a)} \llbracket f_{i}(a)+\operatorname{ap} D f_{i}(a)(v) \rrbracket \text { whenever } v \in \mathbb{R}^{n}
$$

at $\mathcal{L}^{n}$ almost all $a \in A$ where $I(a)=\left\{i \in I: a \in A_{i}\right\}$.

Proof of (1) Since the closure of $f$ in $\mathbb{R}^{n} \times Q_{Q}\left(\mathbb{R}^{m}\right)$ is a Lipschitzian function with the same Lipschitz constant, one may assume $A$ to be closed. Moreover, assume Lip $f>0$ and let $E=\operatorname{graph}_{Q} f, s=\left(1+(\operatorname{Lip} f)^{2}\right)^{-1 / 2}$, and $p: \mathbb{R}^{n} \times \mathbb{R}^{m} \rightarrow \mathbb{R}^{n}, q: \mathbb{R}^{n} \times \mathbb{R}^{m} \rightarrow \mathbb{R}^{m}$ the projections.

If $\xi \in E, 0<2 r \leq \operatorname{dist}(q(\xi),(\operatorname{spt} f(p(\xi))) \sim\{q(\xi)\})$, and $z \in E \cap B_{r}(\xi)$, then

$$
\begin{aligned}
q(\xi) & \in \operatorname{spt} f(p(\xi)), \quad q(z) \in \operatorname{spt} f(p(z)), \\
|q(z)-q(\xi)| & \leq|z-\xi|<r, \quad|q(z)-q(\xi)|=\operatorname{dist}(q(z), \text { spt } f(p(\xi))), \\
|q(z)-q(\xi)| & \leq \mathcal{G}(f(p(z)), f(p(\xi))) \leq(\operatorname{Lip} f)|p(z)-p(\xi)|, \\
|z-\xi| & \leq s^{-1}|p(z)-p(\xi)|, \quad z \notin X(\xi, r, \text { ker } p, s) .
\end{aligned}
$$

Therefore $E$ is the union of the sets

$$
E_{j}:=\{\xi \in E: E \cap X(\xi, 1 / j, \text { ker } p, s)=\emptyset\}
$$

corresponding to $j \in \mathbb{N}$.

Since $E_{j} \subset E$, it follows from the proof of $[6,3.3 .5]$ that each subset of $E_{j}$ with diameter less that $1 / j$ is a Lipschitzian function with Lipschitz constant at most $\left(s^{-2}-1\right)^{1 / 2}=\operatorname{Lip} f$. Using this fact and noting that $\operatorname{graph}_{Q} f$ is closed, one constructs a sequence of closed sets $g_{j}$ with $\operatorname{Lip} g_{j} \leq \operatorname{Lip} f$, here $\operatorname{Lip} \emptyset=0$, and $\bigcup\left\{g_{j}: j \in \mathbb{N}\right\}=\operatorname{graph}_{Q} f$ and defines

$$
h_{j, v}=\left(g_{j} \sim \bigcup_{k<j} g_{k}\right) \cap\left\{(x, y): \theta^{0}(\|f(x)\|, y)=v\right\} \text { for } j \in \mathbb{N}, v=1, \ldots, Q .
$$

Since $\theta^{0}(\|S\|, y)$ depends upper semi continuously on $(y, S) \in \mathbb{R}^{m} \times Q_{Q}\left(\mathbb{R}^{n}\right)$, the functions $h_{j, v}$ and hence $\operatorname{dmn} h_{j, v}$ are Borel sets by [6, 2.2.10(2)]. Arranging $I$ and $f_{i}$ such that each $h_{j, v}$ occurs exactly $v$ times among the $f_{i}$ the conclusion follows.

Proof of (2) For $a \in A$ note that \#I(a)=Q and

$$
f(x)=\sum_{i \in I(a)} \llbracket f_{i}(x) \rrbracket \quad \text { whenever } x \in \bigcap_{i \in I(a)} \operatorname{dmn} f_{i} .
$$


By $[6,2.9 .11,3.1 .2,3.1 .7] \mathcal{L}^{n}$ almost all $a \in A$ satisfy

$i \in I(a)$ implies $f_{i}$ is approximately differentiable at $a$,

$i, j \in I(a), f_{i}(a)=f_{j}(a)$ implies ap $D f_{i}(a)=\operatorname{ap} D f_{j}(a)$.

At such a point $a$ there holds $\theta^{n}\left(\mathcal{L}^{n}\left\llcorner\mathbb{R}^{n} \sim \bigcap_{i \in I(a)} \operatorname{dmn} f_{i}, a\right)=0\right.$, and $f$ is therefore approximately strongly affinely approximable with

$$
\text { ap } A f(a)(v)=\sum_{i \in I(a)} \llbracket f_{i}(a)+\text { ap } D f_{i}(a)(v) \rrbracket \text { for } v \in \mathbb{R}^{n} .
$$

Remark 2.6 Instead of referring to [6, 2.2.10(2)] in the proof of (1), one could have used the more elementary fact that $p(B \sim C)$ is a Borel set whenever $B$ and $C$ are closed subsets of $\mathbb{R}^{n} \times \mathbb{R}^{m}$.

Remark 2.7 In [8, Sect. 5] Goblet gives an example with $n=2, m=2$ and $A$ the unit sphere in $\mathbb{R}^{2}$ such that no continuous function $g: A \rightarrow \mathbb{R}^{m}$ satisfies $g(x) \in \operatorname{spt} f(x)$ whenever $x \in A$. Hence, in general the domain of the functions $f_{i}$ will not equal $A$.

Corollary 2.8 Suppose $m, n, Q \in \mathbb{N}, A \subset B \subset \mathbb{R}^{n}, B$ is open, $f: B \rightarrow Q_{Q}\left(\mathbb{R}^{m}\right)$, and

$$
\limsup _{x \rightarrow a} \mathcal{G}(f(x), f(a)) /|x-a|<\infty \text { whenever } a \in A .
$$

Then $f$ is strongly affinely approximable at $\mathcal{L}^{n}$ almost all points of $A$.

Proof The proof follows closely [6, 3.1.9].

The set $A$ is contained in the union of

$$
C_{j}=B \cap\left\{z: \mathcal{G}(f(x), f(z)) \leq j|x-z| \text { for } x \in B_{1 / j}(z)\right\}
$$

corresponding to $j \in \mathbb{N}$. Verifying as in $[6,3.1 .9]$ that $C_{j}$ is closed, one expresses $C_{j}$ as the union of closed sets $C_{j, 1}, C_{j, 2}, C_{j, 3}, \ldots$ with diameters less than $1 / j$ and notes that $f \mid C_{j, k}$ is Lipschitzian. From Theorem 2.5 and $[6,2.9 .11]$ one infers that at $\mathcal{L}^{n}$ almost all points $x$ of $C_{j, k}$ the function $f \mid C_{j, k}$ is approximately strongly affinely approximable and $\mathbb{R}^{n} \sim C_{j, k}$ has density 0 at $x$, hence $f$ is approximately strongly affinely approximable and $\mathbb{R}^{n} \sim C_{j}$ has density 0 at $x$, hence $f$ is strongly affinely approximable at $x$ by $[6,3.1 .5]$ applied with $f(z)$ replaced by $\mathcal{G}(f(z)$, ap $A f(x)(z))$.

Definition 2.9 Suppose $m, n, Q \in \mathbb{N}, S \in Q_{Q}\left(\mathbb{R}^{m}\right), 1 \leq q \leq \infty, A$ is $\mathcal{L}^{n}$ measurable, and $f: A \rightarrow Q_{Q}\left(\mathbb{R}^{m}\right)$ is an $\mathcal{L}^{n}\llcorner A$ measurable function.

Then the $q$ height of $f$ with respect to $S$ is defined to be the $L^{q}\left(\mathcal{L}^{n}\llcorner A)\right.$ (semi) norm of the function mapping $x \in A$ to $\mathcal{G}(f(x), S)$, denoted by $h_{q}(f, S)$, and, if $f$ is additionally Lipschitzian, then the $q$ tilt of $f$ is defined to be the $L^{q}\left(\mathcal{L}^{n}\llcorner A)\right.$ (semi) norm of the function mapping $x \in A$ to $\mid$ ap $A f(x) \mid$, denoted by $t_{q}(f)$. Moreover, the $q$ height of $f$ is defined to be the infimum of the numbers $h_{q}(f, S)$ corresponding to all $S \in Q_{Q}\left(\mathbb{R}^{m}\right)$ and denoted by $h_{q}(f)$.

Theorem 2.10 Suppose $m, n, Q \in \mathbb{N}, f: \bar{B}_{1}^{n}(0) \rightarrow Q_{Q}\left(\mathbb{R}^{m}\right)$, and Lip $f<\infty$.

Then the following two statements hold: 
(1) If $1 \leq q<n, q^{*}=q n /(n-q)$, then there exists a positive, finite number $\Gamma_{(1)}$ depending only on $m, n, Q$, and $q$ such that

$$
h_{q^{*}}(f) \leq \Gamma_{(1)} t_{q}(f) .
$$

(2) If $n<q \leq \infty$, then there exists a positive, finite number $\Gamma_{(2)}$ depending only on $m, n$, $Q$, and $q$ such that

$$
h_{\infty}(f) \leq \Gamma_{(2)} t_{q}(f) .
$$

Proof Defining $P, \xi: Q_{Q}\left(\mathbb{R}^{m}\right) \rightarrow \mathbb{R}^{P Q}$ and $\rho: \mathbb{R}^{P Q} \rightarrow$ im $\xi$ as in Almgren [2, 1.2(3), 1.3(1)] and noting, using Almgren [2, 1.2(3), 1.3(1), 1.4(3)], that

$$
\begin{aligned}
\xi^{-1} \circ \rho \circ \xi & =\mathbb{1} Q_{Q}\left(\mathbb{R}^{m}\right), \quad \operatorname{Lip} \xi<\infty, \quad \operatorname{Lip} \xi^{-1}<\infty, \quad \operatorname{Lip} \rho<\infty, \\
\mathcal{G}\left(f(x), \xi^{-1}(\rho(z))\right) & \leq \operatorname{Lip} \xi^{-1} \operatorname{Lip} \rho|\xi(f(x))-z| \text { for } x \in \bar{B}_{1}^{n}(0), z \in \mathbb{R}^{P Q}, \\
|D(\xi \circ f)(x)| & \leq \operatorname{Lip} \xi|A f(x)| \text { for } x \in \operatorname{dmn} D(\xi \circ f),
\end{aligned}
$$

the assertion is readily deduced from classical embedding results (which can be deduced for example from [7, Lemma 7.14] using estimates on convolution (cf. O'Neil [12]) for part (1) and Hölder's inequality for part (2)) applied to $\xi \circ f$.

Remark 2.11 The use of Almgren [2, 1.3(1)], i.e. the use of $\varrho$, can be replaced by a more elementary argument, see De Lellis and Spadaro [5, Proposition 2.12].

Theorem 2.12 (cf. [2, 1.3(2)])

Suppose $m, n, Q \in \mathbb{N}, A \subset \mathbb{R}^{n}$, and $f: A \rightarrow Q_{Q}\left(\mathbb{R}^{m}\right)$.

Then there exists $g: \mathbb{R}^{n} \rightarrow Q_{Q}\left(\mathbb{R}^{m}\right)$ such that

$$
g \mid A=f, \quad \operatorname{Lip} g \leq \Gamma \operatorname{Lip} f
$$

where $\Gamma$ is a positive, finite number depending only on $m$ and $Q$.

Definition 2.13 Suppose $m, n, Q \in \mathbb{N}$, and $T \in G(n+m, n)$.

Then $P$ is called a $Q$ valued plane parallel to $T$ if and only if for some $S \in Q_{Q}\left(T^{\perp}\right)$

$$
P=\left(\theta^{0}(\|S\|, \cdot) \circ T^{\perp}\right) \mathcal{H}^{n} .
$$

$S$ is uniquely determined by $P$. For any two $Q$ valued planes $P_{1}$ and $P_{2}$ parallel to $T$ associated to $S_{1}, S_{2} \in Q_{Q}\left(T^{\perp}\right)$ one defines

$$
\mathcal{G}\left(P_{1}, P_{2}\right):=\mathcal{G}\left(S_{1}, S_{2}\right) .
$$

In particular, if $S=\sum_{i=1}^{Q} \llbracket z_{i} \rrbracket$ for some $z_{1}, \ldots, z_{Q} \in T^{\perp}$, then

$$
\|S\|=\sum_{i=1}^{Q} \delta_{z_{i}}, \quad P=\sum_{i=1}^{Q} \mathcal{H}^{n}\left\llcorner\left\{x \in \mathbb{R}^{n+m}: T^{\perp}(x)=z_{i}\right\}\right.
$$

where $\delta_{x}$ denotes the Dirac measure at the point $x$.

2.14 If $0<d<\infty, m \in \mathbb{N}, S, T \in Q_{Q}\left(\mathbb{R}^{m}\right)$, and for each subset $X$ of spt $S$

$$
\sum_{x \in X} \theta^{0}(\|S\|, x)+\sum_{y \in Y} \theta^{0}(\|T\|, y) \leq Q
$$


where $Y=(\operatorname{spt} T) \sim \bigcup_{x \in X} B_{d}(x)$, then

$$
\mathcal{G}(S, T)<Q^{1 / 2} d
$$

in fact if $S=\sum_{i=1}^{Q} \llbracket x_{i} \rrbracket, T=\sum_{i=1}^{Q} \llbracket y_{i} \rrbracket$ for some $x_{1}, \ldots, x_{Q}, y_{1}, \ldots, y_{Q} \in \mathbb{R}^{m}$ one may verify the existence of a permutation $\sigma$ of $\{1, \ldots, Q\}$ such that $\left|x_{i}-y_{\sigma(i)}\right|<d$ for $i \in\{1, \ldots, Q\}$ by Hall's theorem on perfect matches, see e.g. [9, Theorem 1.1.3].

\section{Approximation of integral varifolds}

In this section an approximation procedure for integral $n$ varifolds $\mu$ in $\mathbb{R}^{n+m}$ by $Q$ valued functions is carried out. Similar constructions are used in [2, 3.1-3.12] by Almgren and in $[4,5.4]$ by Brakke. Basically, a part of $\mu$ which is suitably close to a $Q$ valued plane is approximated "above" a subset $Y$ of $\mathbb{R}^{n}$ by a Lipschitzian $Q$ valued function. The sets where this approximation fails are estimated in terms of both $\mu$ and $\mathcal{L}^{n}$ measure.

Taking Brakke's version as a starting point, in order to obtain an approximation useful for proving Theorems 4.4 and 4.11 in the next section, the following three problems had to be solved.

Firstly, in the above mentioned estimate one can only allow for tilt and mean curvature terms and not for a height term as it is present in Brakke $[4,5.4]$. This is done using a new version of Brakke's multilayer monotonicity in $[4,5.3]$ which allows for variable offsets, see Lemma 3.11.

Secondly, the seemingly most natural way to estimate the height of $\mu$ above the complement of $Y$, namely measure times maximal height $h$, would not produce sharp enough an estimate. In order to circumvent this difficulty, a "preliminary graphical part" $H$ of $\mu$ is used which is larger than the part where $\mu$ equals the "graph" of the $Q$ valued function and also slightly larger than the "graphical part" $G$ defined in terms of mean curvature used in the statement of Theorem 4.4. Points in $H$ still satisfy a one sided Lipschitz condition with respect to points above $Y$, see Lemmas 3.12(2) and 3.15(4). Using this fact in conjunction with a covering argument in Lemma 3.15(6) the actual error in estimating the $q$ height in a ball $\bar{B}_{t}(\zeta)$ where $\mathcal{L}^{n}\left(\bar{B}_{t}(\zeta) \cap Y\right)$ and $\mathcal{L}^{n}\left(\bar{B}_{t}(\zeta) \sim Y\right)$ are comparable, can be estimated by $\mathcal{L}^{n}\left(\bar{B}_{t}(\zeta) \sim Y\right)^{1 / q} \cdot t$ instead of $\mathcal{L}^{n}\left(\bar{B}_{t}(\zeta) \sim Y\right)^{1 / q} \cdot h$; the replacement of $h$ by $t$ being the decisive improvement which allows to estimate the $q^{*}$ height $\left(q^{*}=n q /(n-q), 1 \leq q<n\right)$ instead of the $q$ height in Theorem 4.4.

Thirdly, to obtain a sharp result with respect to the assumptions on the mean curvature, all curvature conditions are phrased in terms of isoperimetric ratios in order to allow for the application of the estimates in [11]. In this situation it seems to be impossible to derive monotonicity results from the monotonicity formula (cf. [16, (17.3)]). Instead, it is shown that nonintegral bounds for density ratios are preserved provided the varifold is additionally close to a plane, see Lemma 3.9. The latter result appears to be generally useful in deriving sharp estimates involving mean curvature.

Comparing the present construction to Almgren's, one notes that his version does not contain a height term and establishes the important one sided Lipschitz condition in [2, 3.8 (4)]. However, both properties are proven only under a $L^{\infty}$ smallness condition on the mean curvature. Almgren uses an elaborate inductive construction obtaining explicit estimates by use of the monotonicity identity in Allard [1, 5.1(1)]. These estimates provide quantitative control of the effect of prescribing a small Lipschitz constant for the approximating function on the accuracy of the approximation in mass; a feature which is apparently important for 
the applications in the course of that paper. Since such kind of control is not needed here and since explicit estimates cannot be easily derived from the present rather weak conditions on the mean curvature, contradiction arguments in Lemma 3.11 and Lemma 3.12 together with the identification of the "preliminary graphical part" are used to establish the afore-mentioned two properties of Almgren's construction in the present setting. In fact, even in the case of multiplicity 1 , deriving explicit estimates is connected to determining the best value in the isoperimetric inequality, see $[11,2.4-6]$.

3.1 If $m, n \in \mathbb{N}, a \in \mathbb{R}^{n+m}, 0<r<\infty, T \in G(n+m, n)$, and $\mu$ is a stationary, integral $n$ varifold in $B_{r}(a)$ with $T_{x} \mu=T$ for $\mu$ almost all $x \in B_{r}(a)$, then $T^{\perp}$ (spt $\mu$ ) is discrete and closed in $T^{\perp}\left(B_{r}(a)\right)$ and for every $x \in \operatorname{spt} \mu$

$$
y \in B_{r}(a), y-x \in T \text { implies } \theta^{n}(\mu, y)=\theta^{n}(\mu, x) \in \mathbb{N} ;
$$

hence with $S_{x}=\left\{y \in B_{r}(a): y-x \in T\right\}$

$$
\mu\left\llcorner S_{x}=\theta^{n}(\mu, x) \mathcal{H}^{n}\left\llcorner S_{x} \quad \text { whenever } x \in B_{r}(a) .\right.\right.
$$

A similar assertion may be found in Almgren [2,3.6] and is used by Brakke in [4, 5.3(16)].

Lemma 3.2 Suppose $m, n \in \mathbb{N}, 0<\delta<1,0 \leq s<1$, and $0 \leq M<\infty$.

Then there exists a positive, finite number $\varepsilon$ with the following property.

If $a \in \mathbb{R}^{n+m}, 0<r<\infty, T \in G(n+m, n), 0 \leq d<\infty, 0<t<\infty, \zeta \in \mathbb{R}^{n+m}$,

$$
\max \{d, r\} \leq M t, \quad \zeta \in \bar{B}_{d}^{n+m}(0) \cap T, \quad d+t \leq r,
$$

$\mu$ is an integral $n$ varifold in $B_{r}(a)$ with locally bounded first variation,

$$
\begin{aligned}
\|\delta \mu\|\left(B_{r}(a)\right) & \leq \varepsilon \mu\left(B_{r}(a)\right)^{1-1 / n}, \quad \mu\left(B_{r}(a)\right) \leq M \omega_{n} r^{n}, \\
\int_{B_{r}(a)}\left|T_{\xi} \mu-T\right| \mathrm{d} \mu(\xi) & \leq \varepsilon \mu\left(B_{r}(a)\right), \\
\mu\left(\bar{B}_{\varrho}(a)\right) & \geq \delta \omega_{n} \varrho^{n} \text { for } 0<\varrho<r,
\end{aligned}
$$

then

$$
\mu\left(\left\{x \in B_{t}(a+\zeta):|T(x-a)|>s|x-a|\right\}\right) \geq(1-\delta) \omega_{n} t^{n} .
$$

Proof If the lemma were false for some $m, n \in \mathbb{N}, 0<\delta<1,0 \leq s<1$, and $0 \leq M<\infty$ there would exist a sequence $\varepsilon_{i}$ with $\varepsilon_{i} \downarrow 0$ as $i \rightarrow \infty$ and sequences $a_{i}, r_{i}, T_{i}, d_{i}, t_{i}, \zeta_{i}$, and $\mu_{i}$ showing that $\varepsilon_{i}$ does not satisfy the conclusion of the lemma.

One could assume for some $T \in G(n+m, n)$, using isometries and homotheties,

$$
T_{i}=T, \quad r_{i}=1, \quad a_{i}=0
$$

for $i \in \mathbb{N}$. Therefore passing to a subsequence, there would exist $0 \leq d<\infty, 0 \leq t<\infty$, $\zeta_{i} \in \mathbb{R}^{n+m}$ such that

$$
d_{i} \rightarrow d, \quad t_{i} \rightarrow t, \quad \zeta_{i} \rightarrow \zeta
$$

as $i \rightarrow \infty$. There would hold

$$
\max \{d, 1\} \leq M t, \quad \zeta \in \bar{B}_{d}^{n+m}(0) \cap T, \quad d+t \leq 1,
$$


in particular $t>0$. Possibly passing to another subsequence, one could construct (cf. Allard $[1,6.4])$ a stationary, integral $n$ varifold $\mu$ in $B_{1}^{n+m}(0)$ with

$$
T_{x} \mu=T \text { for } \mu \text { almost all } x \in B_{1}^{n+m}(0)
$$

such that

$$
\int \phi \mathrm{d} \mu_{i} \rightarrow \int \phi \mathrm{d} \mu \text { for } i \rightarrow \infty \text { for } \phi \in C_{\mathrm{c}}^{0}\left(B_{1}^{n+m}(0)\right) .
$$

Since any open subset of $\mathbb{R}^{n+m}$ with compact closure in $\left\{x \in B_{t}(\zeta):|T(x)|>s|x|\right\}$ would be contained in $\left\{x \in B_{t_{i}}\left(\zeta_{i}\right):|T(x)|>s|x|\right\}$ for large $i$, one could estimate

$$
\begin{aligned}
& \mu\left(\left\{x \in B_{t}(\zeta):|T(x)|>s|x|\right\}\right) \\
& \quad \leq \liminf _{i \rightarrow \infty} \mu_{i}\left(\left\{x \in B_{t_{i}}\left(\zeta_{i}\right):|T(x)|>s|x|\right\}\right) \leq(1-\delta) \omega_{n} t^{n} .
\end{aligned}
$$

This would imply by 3.1 that $0 \notin \operatorname{spt} \mu$ in contradiction to

$$
\mu\left(\bar{B}_{\varrho}^{n+m}(0)\right) \geq \limsup _{i \rightarrow \infty} \mu_{i}\left(\bar{B}_{\varrho}^{n+m}(0)\right) \geq \delta \omega_{n} \varrho^{n} \text { for } 0<\varrho<1 .
$$

Definition 3.3 Whenever $n \in \mathbb{N}$ the symbol $\gamma_{n}$ will denote the smallest number with the following property:

If $m \in \mathbb{N}_{0}$ and $\mu$ is a rectifiable $n$ varifold in $\mathbb{R}^{n+m}$ with $\mu\left(\mathbb{R}^{n+m}\right)<\infty$ and $\|\delta \mu\|\left(\mathbb{R}^{n+m}\right)<\infty$, then

$$
\mu\left(\left\{x \in \mathbb{R}^{n+m}: \theta^{n}(\mu, x) \geq 1\right\}\right) \leq \gamma_{n} \mu\left(\mathbb{R}^{n+m}\right)^{1 / n}\|\delta \mu\|\left(\mathbb{R}^{n+m}\right) .
$$

Properties of this number are given in [11, Sect. 2], in particular $\gamma_{n}<\infty$; see also Allard [1, Theorem 7.1] or [16, Lemma 18.7, Theorem 18.6].

Lemma 3.4 (cf. $[11,2.6]$ ) Suppose $m \in \mathbb{N}_{0}, n \in \mathbb{N}$, and $\delta>0$.

Then there exists a positive number $\varepsilon$ with the following property.

If $a \in \mathbb{R}^{n+m}, 0<r<\infty, \mu$ is a rectifiable $n$ varifold in $B_{r}(a)$ of locally bounded first variation such that $\theta^{n}(\mu, x) \geq 1$ for $\mu$ almost all $x \in B_{r}(a), a \in \operatorname{spt} \mu$, and

$$
\begin{aligned}
\|\delta \mu\|\left(\bar{B}_{\varrho}(a)\right) \leq & \left(2 \gamma_{n}\right)^{-1} \mu\left(\bar{B}_{\varrho}(a)\right)^{1-1 / n} \text { for } 0<\varrho<r, \\
& \|\delta \mu\|\left(B_{r}(a)\right) \leq \varepsilon \mu\left(B_{r}(a)\right)^{1-1 / n},
\end{aligned}
$$

then

$$
\mu\left(B_{r}(a)\right) \geq(1-\delta) \omega_{n} r^{n}
$$

3.5 Suppose $-\infty<a<b<\infty, I=[a, b], f: I \rightarrow \mathbb{R}$ is nondecreasing and continuous from the left, $g: I \rightarrow \mathbb{R}$ is continuous, and $f(a) \geq g(a), f(b)<g(b)$.

Then there exists $\xi$ with $a \leq \xi<b$ such that

$$
f(\xi)=g(\xi), \quad \text { and } \quad f(t) \geq g(t) \text { whenever } \xi \geq t \in I ;
$$

in fact one may take $\xi=\inf \{t \in I: f(t)<g(t)\}$.

Lemma 3.6 (Multilayer monotonicity) Suppose $m, n, Q \in \mathbb{N}, 0<\delta \leq 1$, and $0 \leq s<1$.

Then there exists a positive, finite number $\varepsilon$ with the following property. 
If $X \subset \mathbb{R}^{n+m}, T \in G(n+m, n), 0<r<\infty$,

$$
|T(y-x)| \leq s|y-x| \text { whenever } x, y \in X,
$$

$\mu$ is an integral $n$ varifold in $\bigcup_{x \in X} B_{r}(x)$ with locally bounded first variation,

$$
\sum_{x \in X} \theta_{*}^{n}(\mu, x) \geq Q-1+\delta
$$

and whenever $0<\varrho<r, x \in X \cap \operatorname{spt} \mu$

$$
\|\delta \mu\|\left(\bar{B}_{\varrho}(x)\right) \leq \varepsilon \mu\left(\bar{B}_{\varrho}(x)\right)^{1-1 / n}, \quad \int_{\bar{B}_{\varrho}(x)}\left|T_{\xi} \mu-T\right| \mathrm{d} \mu(\xi) \leq \varepsilon \mu\left(\bar{B}_{\varrho}(x)\right),
$$

then

$$
\mu\left(\bigcup_{x \in X} B_{\varrho}(x)\right) \geq(Q-\delta) \omega_{n} \varrho^{n} \quad \text { whenever } 0<\varrho \leq r
$$

Proof The proof follows closely Brakke $[4,5.3]$.

If the lemma were false for some $m, n, Q \in \mathbb{N}, 0<\delta<1 / 2$, and $0<s<1$, there would exist a sequence $\varepsilon_{i}$ with $\varepsilon_{i} \downarrow 0$ as $i \rightarrow \infty$ and sequences $X_{i}, T_{i}, r_{i}$, and $\mu_{i}$ showing that $\varepsilon_{i}$ does not satisfy the conclusion of the lemma.

Clearly, one could assume for some $T \in G(n+m, n)$

$$
T_{i}=T \quad \text { for } i \in \mathbb{N},
$$

$X_{i} \subset \operatorname{spt} \mu_{i}$ for $i \in \mathbb{N}$, and in view of Lemma 3.4 also

$$
\# X_{i} \leq Q \text { for } i \in \mathbb{N} .
$$

One would observe that 3.5 could be used to deduce the existence of a sequence $0<\varrho_{i}<r_{i}$ such that

$$
\begin{aligned}
& \mu_{i}\left(\bigcup_{x \in X_{i}} B_{\varrho_{i}}(x)\right) \leq(Q-\delta) \omega_{n}\left(\varrho_{i}\right)^{n}, \\
& \mu_{i}\left(\bigcup_{x \in X_{i}} B_{\varrho}(x)\right) \geq(Q-1+\delta / 2) \omega_{n} \varrho^{n} \quad \text { whenever } 0<\varrho \leq \varrho_{i} .
\end{aligned}
$$

There would hold for $x \in X_{i}, i \in \mathbb{N}$

$$
\begin{gathered}
\left\|\delta \mu_{i}\right\|\left(B_{\varrho_{i}}(x)\right) \leq \varepsilon_{i}\left(Q \omega_{n}\right)^{1-1 / n}\left(\varrho_{i}\right)^{n-1}, \\
\int_{B_{\varrho_{i}}(x)}\left|T_{\xi} \mu_{i}-T\right| \mathrm{d} \mu_{i}(\xi) \leq \varepsilon_{i} Q \omega_{n}\left(\varrho_{i}\right)^{n} .
\end{gathered}
$$

Rescaling, one would infer the existence of sequences of integral $n$ varifolds $v_{i}$ in $\mathbb{R}^{n+m}$, $X_{i} \subset$ spt $v_{i}$, and $\varepsilon_{i}$ with $\varepsilon_{i} \downarrow 0$ as $i \rightarrow \infty$ such that for some $T \in G(n+m, n), 0<M<\infty$, $Q \in \mathbb{N}, 0<\delta<1 / 2$, and $0<s<1$ 


$$
\begin{aligned}
\# X_{i} & \leq Q, \quad s^{-1}|T(y-x)| \leq|y-x| \text { for } x, y \in X_{i}, \\
\left\|\delta v_{i}\right\|\left(B_{1}(x)\right) & \leq \varepsilon_{i} M, \int_{B_{1}(x)}\left|T_{\xi} v_{i}-T\right| \mathrm{d} v_{i}(\xi) \leq \varepsilon_{i} M \text { for } x \in X_{i}, \\
v_{i}\left(\bigcup_{x \in X_{i}} B_{1}(x)\right) & \leq(Q-\delta) \omega_{n}, \\
v_{i}\left(\bigcup_{x \in X_{i}} B_{\varrho}(x)\right) & \geq(Q-1+\delta / 2) \omega_{n} \varrho^{n} \quad \text { whenever } 0<\varrho \leq 1 .
\end{aligned}
$$

The proof will be concluded by showing that objects with the properties described in the preceding paragraph do not exist. If they existed, one could assume first

$$
X_{i} \subset \bar{B}_{M}^{n+m}(0) \text { for } i \in \mathbb{N}
$$

by moving pieces of $v_{i}$ by translations (here $v$ is a piece of $v_{i}$ if and only if $v=v_{i}\llcorner Z$ for some connected component $Z$ of $\left.\bigcup_{x \in X_{i}} B_{1}(x)\right)$ and then, since $X_{i} \neq \emptyset$ for $i \in \mathbb{N}$, passing to a subsequence,

$$
X_{i} \rightarrow X \quad \text { in Hausdorff distance as } i \rightarrow \infty, \quad \# X \leq Q
$$

for some nonempty, closed subset $X$ of $\bar{B}_{M}^{n+m}(0)$ (cf. [6, 2.10.21]). Noting that given $0<$ $\varrho_{1}<\varrho_{2}<1$

$$
\bigcup_{x \in X} B_{\varrho_{1}}(x) \subset \bigcup_{x \in X_{i}} B_{\varrho_{2}}(x), \bigcup_{x \in X} B_{\varrho_{2}}(x) \supset \bigcup_{x \in X_{i}} B_{\varrho_{1}}(x)
$$

for large $i$, one could assume, possibly passing to another subsequence (cf. Allard [1, 6.4]), that for some stationary, integral $n$ varifold $v$ in

$$
U:=\bigcup_{x \in X} B_{1}(x)
$$

satisfying

$$
T_{x} v=T \text { for } v \text { almost all } x \in U
$$

there would hold

$$
\int \varphi \mathrm{d} v_{i} \rightarrow \int \varphi \mathrm{d} v \quad \text { as } i \rightarrow \infty \text { for } \varphi \in C_{\mathrm{c}}^{0}\left(\mathbb{R}^{n+m}\right) \text { with } \operatorname{spt} \varphi \subset U .
$$

The inclusions previously noted, would show

$$
\begin{aligned}
v(U) & \leq(Q-\delta) \omega_{n}, \\
v\left(\bigcup_{x \in X} B_{\varrho}(x)\right) & \geq(Q-1+\delta / 2) \omega_{n} \varrho^{n} \quad \text { for } 0<\varrho \leq 1 .
\end{aligned}
$$

Since for $y, z \in X$

$$
\begin{gathered}
s^{-1}|T(y-x)| \leq|y-x|, \\
\left\{x \in \mathbb{R}^{n+m}: y-x \in T\right\} \cap\left\{x \in \mathbb{R}^{n+m}: z-x \in T\right\}=\emptyset \text { if } y \neq z,
\end{gathered}
$$


these inequalities would imply by 3.1

$$
\begin{aligned}
Q-1+\delta / 2 & \leq \liminf _{\varrho \downarrow 0} v\left(\bigcup_{x \in X} B_{\varrho}(x)\right) /\left(\omega_{n} \varrho^{n}\right) \\
& =\sum_{x \in X} \theta^{n}(v, x) \leq v(U) / \omega_{n} \leq Q-\delta ;
\end{aligned}
$$

a contradiction to $\sum_{x \in X} \theta^{n}(v, x) \in \mathbb{N}$.

Lemma 3.7 Suppose $0<M<\infty, M \notin \mathbb{N}, 0<\lambda_{1}<\lambda_{2}<1, m, n \in \mathbb{N}, T \in G(n+m, n)$, $F$ is the family of all stationary, integral $n$ varifolds in $B_{1}^{n+m}(0)$ such that

$$
T_{x} \mu=T \text { for } \mu \text { almost all } x \in B_{1}^{n+m}(0), \quad \mu\left(B_{1}^{n+m}(0)\right) \leq M \omega_{n},
$$

and $N$ is the supremum of all numbers

$$
\left(\omega_{n} r^{n}\right)^{-1} \mu\left(\bar{B}_{r}^{n+m}(0)\right)
$$

corresponding to all $\mu \in F$ and $\lambda_{1} \leq r \leq \lambda_{2}$.

Then for some $\mu \in F$ and some $\lambda_{1} \leq r \leq \lambda_{2}$

$$
N=\left(\omega_{n} r^{n}\right)^{-1} \mu\left(\bar{B}_{r}^{n+m}(0)\right)<M .
$$

Proof The proof uses the structure of the elements of $F$ described in 3.1. Since

$$
\left(\omega_{n} r^{n}\right)^{-1} \mu\left(\bar{B}_{r}^{n+m}(0)\right)
$$

depends continuously on $(\mu, r) \in F \times\left[\lambda_{1}, \lambda_{2}\right]$, the first part of the conclusion is a consequence of the fact that $F$ is compact with respect to the weak topology by Allard $[1,6.4]$. To prove the second part, one notes

$$
\left(r^{2}-\varrho^{2}\right)^{n / 2}<\left(1-\varrho^{2}\right)^{n / 2} r^{n} \quad \text { whenever } 0<\varrho \leq r<1,
$$

defines $\Theta: T^{\perp}(\operatorname{spt} \mu) \rightarrow \mathbb{N}$ such that $\Theta \circ T^{\perp} \mid B_{1}^{n+m}(0)=\theta^{n}(\mu, \cdot)$ and computes

$$
\begin{aligned}
\mu\left(\bar{B}_{r}^{n+m}(0)\right) & =\sum_{x \in T^{\perp}\left(\bar{B}_{r}^{n+m}(0) \cap \operatorname{spt} \mu\right)} \Theta(x) \omega_{n}\left(r^{2}-|x|^{2}\right)^{n / 2} \\
& \leq\left(\sum_{x \in T^{\perp}\left(\bar{B}_{r}^{n+m}(0) \cap \operatorname{spt} \mu\right)} \Theta(x) \omega_{n}\left(1-|x|^{2}\right)^{n / 2}\right) r^{n} \\
& \leq \mu\left(B_{1}^{n+m}(0)\right) r^{n} \leq M \omega_{n} r^{n} .
\end{aligned}
$$

If spt $\mu \not \subset T$, then the first or the second inequality in the computation is strict. Otherwise, the last inequality is strict because $M \notin \mathbb{N}$.

Remark 3.8 Alternately, the second part can be obtained by use of the monotonicity formula (cf. $[16,(17.5)])$.

Lemma 3.9 (Quasi monotonicity) Suppose $0<M<\infty, M \notin \mathbb{N}, 0<\lambda<1$, and $m, n \in \mathbb{N}$.

Then there exists a positive, finite number $\varepsilon$ with the following property.

If $a \in \mathbb{R}^{n+m}, 0<r<\infty, \mu$ is an integral $n$ varifold in $B_{r}(a)$ with locally bounded first variation,

$$
\mu\left(B_{r}(a)\right) \leq M \omega_{n} r^{n}
$$


and whenever $0<\varrho<r$

$$
\begin{aligned}
\|\delta \mu\|\left(\bar{B}_{\varrho}(a)\right) & \leq \varepsilon \mu\left(\bar{B}_{\varrho}(a)\right)^{1-1 / n}, \\
\int_{\bar{B}_{\varrho}(a)}\left|T_{x} \mu-T\right| \mathrm{d} \mu(x) & \leq \varepsilon \mu\left(\bar{B}_{\varrho}(a)\right) \text { for some } T \in G(n+m, n),
\end{aligned}
$$

(here $0^{0}:=1$ ), then

$$
\mu\left(\bar{B}_{\varrho}(a)\right) \leq M \omega_{n} \varrho^{n} \text { whenever } 0<\varrho \leq \lambda r .
$$

Proof Using induction, one verifies that it is enough to prove the statement with $\lambda^{2} r \leq \varrho \leq$ $\lambda r$ replacing $0<\varrho \leq \lambda r$ in the last line which is readily accomplished by a contradiction argument using Lemma 3.7 and Allard's compactness theorem for integral varifolds $[1,6.4]$.

Remark 3.10 Note that in any case $\mu\left(B_{r}(a)\right) \leq M \omega_{n} r^{n}$ implies

$$
\left(\omega_{n} \varrho^{n}\right)^{-1} \mu\left(\bar{B}_{\varrho}(a)\right) \leq M \lambda^{-n} \text { whenever } \lambda r \leq \varrho<r .
$$

Lemma 3.11 (Multilayer monotonicity with variable offset) Suppose $m, n, Q \in \mathbb{N}$, $0 \leq$ $M<\infty, \delta>0$, and $0 \leq s<1$.

Then there exists a positive, finite number $\varepsilon$ with the following property.

If $X \subset \mathbb{R}^{n+m}, T \in G(n+m, n), 0 \leq d<\infty, 0<r<\infty, 0<t<\infty, f: X \rightarrow \mathbb{R}^{n+m}$,

$$
\begin{aligned}
& |T(y-x)| \leq s|y-x|, \quad|T(f(y)-f(x))| \leq s|f(y)-f(x)|, \\
& f(x)-x \in \bar{B}_{d}^{n+m}(0) \cap T, \quad d \leq M t, \quad d+t \leq r
\end{aligned}
$$

for $x, y \in X, \mu$ is an integral $n$ varifold in $\bigcup_{x \in X} B_{r}(x)$ with locally bounded first variation,

$$
\sum_{x \in X} \theta_{*}^{n}(\mu, x) \geq Q-1+\delta, \quad \mu\left(B_{r}(x)\right) \leq M \omega_{n} r^{n} \text { for } x \in X \cap \operatorname{spt} \mu,
$$

and whenever $0<\varrho<r, x \in X \cap \operatorname{spt} \mu$

$$
\|\delta \mu\|\left(\bar{B}_{\varrho}(x)\right) \leq \varepsilon \mu\left(\bar{B}_{\varrho}(x)\right)^{1-1 / n}, \int_{\bar{B}_{\varrho}(x)}\left|T_{\xi} \mu-T\right| \mathrm{d} \mu(\xi) \leq \varepsilon \mu\left(\bar{B}_{\varrho}(x)\right),
$$

then

$$
\mu\left(\bigcup_{x \in X}\left\{y \in B_{t}(f(x)):|T(y-x)|>s|y-x|\right\}\right) \geq(Q-\delta) \omega_{n} t^{n} .
$$

Proof If the lemma were false for some $m, n, Q \in \mathbb{N}, 0 \leq M<\infty, 0<\delta<1$, and $0<s<1$, there would exist a sequence $\varepsilon_{i}$ with $\varepsilon_{i} \downarrow 0$ as $i \rightarrow \infty$ and sequences $X_{i}, T_{i}, d_{i}$, $r_{i}, t_{i}, f_{i}$, and $\mu_{i}$ showing that $\varepsilon_{i}$ does not satisfy the conclusion of the lemma.

In view of Lemma 3.9 and Remark 3.10 one could assume $d_{i}+t_{i}=r_{i}$ for $i \in \mathbb{N}$ by replacing $M$ by $2 M$. Using isometries and homotheties, one could also assume for some $T \in G(n+m, n)$

$$
T_{i}=T, \quad r_{i}=1
$$

for $i \in \mathbb{N}$. Finally, one could assume, possibly replacing $M$ by a larger number,

$$
X_{i} \subset \operatorname{spt} \mu_{i}, \quad \# X_{i} \leq Q, \quad X_{i} \subset \bar{B}_{M}^{n+m}(0)
$$

for $i \in \mathbb{N}$. 
Therefore passing to a subsequence (cf. [6, 2.10.21]), there would exist a nonempty, closed subset $X$ of $\bar{B}_{M}^{n+m}(0), 0 \leq d<\infty, 0 \leq t<\infty$, and a nonempty, closed subset $f$ of $\mathbb{R}^{n+m} \times \mathbb{R}^{n+m}$ such that $\# X \leq Q$,

$$
\begin{aligned}
& d_{i} \rightarrow d \text { and } t_{i} \rightarrow t \text { as } i \rightarrow \infty, \\
& X_{i} \rightarrow X \text { and } f_{i} \rightarrow f \text { in Hausdorff distance as } i \rightarrow \infty .
\end{aligned}
$$

There would hold

$$
s^{-1}|T(y-x)| \leq|y-x| \text { for } x, y \in X, \quad d \leq M t, \quad d+t=1, \quad t>0 .
$$

Moreover, since

$$
\left(1-s^{2}\right)^{1 / 2}\left|y_{i}-x_{i}\right| \leq\left|T^{\perp}\left(y_{i}-x_{i}\right)\right|=\left|T^{\perp}\left(f_{i}\left(y_{i}\right)-f_{i}\left(x_{i}\right)\right)\right| \leq\left|f_{i}\left(y_{i}\right)-f_{i}\left(x_{i}\right)\right|
$$

for $x_{i}, y_{i} \in X_{i}$, and $i \in \mathbb{N}, f$ were a function and one could readily verify $\operatorname{dmn} f=X$, and

$$
\begin{aligned}
& f(x)-x \in \bar{B}_{d}^{n+m}(0) \cap T \text { for } x \in X, \\
& s^{-1}|T(f(y)-f(x))| \leq|f(y)-f(x)| \text { for } x, y \in X .
\end{aligned}
$$

Possibly passing to another subsequence, one could construct (cf. Allard [1, 6.4]) a stationary, integral $n$ varifold $\mu$ in $U:=\bigcup_{x \in X} B_{1}(x)$ with

$$
T_{x} \mu=T \text { for } \mu \text { almost all } x \in U
$$

such that

$$
\int \varphi \mathrm{d} \mu_{i} \rightarrow \int \varphi \mathrm{d} \mu \quad \text { as } i \rightarrow \infty \text { for } \varphi \in C_{\mathrm{c}}^{0}\left(\mathbb{R}^{n+m}\right) \text { with spt } \varphi \subset U .
$$

According to Lemma 3.6 one would estimate for large $i$

$$
\mu_{i}\left(\bigcup_{x \in X_{i}} B_{\varrho}(x)\right) \geq(Q-\delta) \omega_{n} \varrho^{n} \quad \text { whenever } 0<\varrho \leq 1,
$$

hence

$$
\mu\left(\bigcup_{x \in X} B_{\varrho}(x)\right) \geq(Q-\delta) \omega_{n} \varrho^{n} \quad \text { whenever } 0<\varrho \leq 1 .
$$

Therefore, passing to the limit $\varrho \downarrow 0$, one would infer the lower bound (noting 3.1)

$$
\sum_{x \in X} \theta^{n}(\mu, x) \geq Q-\delta
$$

For $y, z \in \mathbb{R}^{n+m}, 0<\varrho<\infty$ define $V(y, z, \varrho)$ to be the set of all $x \in B_{\varrho}(z)$ such that $s^{-1}|T(y-x)|>|y-x|$, and note that every open subset of $\mathbb{R}^{n+m}$ with compact closure in $\bigcup_{x \in X} V(x, f(x), t)$ would be contained in $\bigcup_{x \in X_{i}} V\left(x, f_{i}(x), t_{i}\right)$ for large $i$; hence

$$
\mu\left(\bigcup_{x \in X} V(x, f(x), t)\right) \leq \liminf _{i \rightarrow \infty} \mu_{i}\left(\bigcup_{x \in X_{i}} V\left(x, f_{i}(x), t_{i}\right)\right) \leq(Q-\delta) \omega_{n} t^{n}
$$

On the other hand 3.1 would imply in conjunction with the fact

$$
\left\{x \in \mathbb{R}^{n+m}: x-y \in T\right\} \cap\left\{x \in \mathbb{R}^{n+m}: x-z \in T\right\}=\emptyset
$$


for $y, z \in X$ with $y \neq z$ and the lower bound previously derived

$$
\mu\left(\bigcup_{x \in X} V(x, f(x), t)\right) \geq\left(\sum_{x \in X} \theta^{n}(\mu, x)\right) \omega_{n} t^{n} \geq(Q-\delta) \omega_{n} t^{n},
$$

hence $\sum_{x \in X} \theta^{n}(\mu, x)=Q-\delta$ which is incompatible with $Q-\delta \notin \mathbb{N}$.

Lemma 3.12 Suppose $m, n, Q \in \mathbb{N}, 0<\delta_{1} \leq 1,0<\delta_{2} \leq 1,0 \leq s<1,0 \leq s_{0}<1$, $0 \leq M<\infty$, and $0<\lambda<1$ is uniquely defined by the requirement

$$
\left(1-\lambda^{2}\right)^{n / 2}=\left(1-\delta_{2}\right)+\left(\frac{\left(s_{0}\right)^{2}}{1-\left(s_{0}\right)^{2}}\right)^{n / 2} \lambda^{n} .
$$

Then there exists a positive, finite number $\varepsilon$ with the following property.

If $X \subset \mathbb{R}^{n+m}, T \in G(n+m, n), 0 \leq d<\infty, 0<r<\infty, 0<t<\infty, \zeta \in \mathbb{R}^{n+m}$,

$$
\# T(X)=1, \quad \zeta \in \bar{B}_{d}^{n+m}(0) \cap T, \quad d \leq M t, \quad d+t \leq r,
$$

$\mu$ is an integral $n$ varifold in $\bigcup_{x \in X} B_{r}(x)$ with locally bounded first variation,

$$
\begin{aligned}
& \theta^{n}(\mu, x) \in \mathbb{N} \text { for } x \in X, \\
& \sum_{x \in X} \theta^{n}(\mu, x)=Q, \quad \mu\left(B_{r}(x)\right) \leq M \omega_{n} r^{n} \text { for } x \in X,
\end{aligned}
$$

and whenever $0<\varrho<r, x \in X$

$$
\|\delta \mu\|\left(\bar{B}_{\varrho}(x)\right) \leq \varepsilon \mu\left(\bar{B}_{\varrho}(x)\right)^{1-1 / n}, \quad \int_{\bar{B}_{\varrho}(x)}\left|T_{\xi} \mu-T\right| \mathrm{d} \mu(\xi) \leq \varepsilon \mu\left(\bar{B}_{\varrho}(x)\right)
$$

satisfying

$$
\mu\left(\bigcup_{x \in X}\left\{y \in B_{t}(x+\zeta):|T(y-x)|>s_{0}|y-x|\right\}\right) \leq\left(Q+1-\delta_{2}\right) \omega_{n} t^{n},
$$

then the following two statements hold:

(1) If $0<\tau \leq \lambda t$, then

$$
\mu\left(\bigcup_{x \in X} \bar{B}_{\tau}(x)\right) \leq\left(Q+\delta_{1}\right) \omega_{n} \tau^{n}
$$

(2) If $\xi \in \mathbb{R}^{n+m}$ with $\operatorname{dist}(\xi, X) \leq \lambda t / 2$ and

$$
\mu\left(\bar{B}_{\varrho}(\xi)\right) \geq \delta_{1} \omega_{n} \varrho^{n} \text { for } 0<\varrho<\delta_{1} \operatorname{dist}(\xi, X),
$$

then for some $x \in X$

$$
|T(\xi-x)| \geq s|\xi-x|
$$

Proof of (1) One may first assume $\max \left\{\delta_{1}, \delta_{2}\right\} \leq 1 / 2$ and then $\lambda^{2} \leq \tau / t \leq \lambda$ by iteration of the result observing that the remaining assertion implies inductively

$$
\mu\left(\bigcup_{x \in X} \bar{B}_{\lambda^{-i} \tau}(x)\right) \leq\left(Q+\delta_{1}\right) \omega_{n}\left(\lambda^{-i} \tau\right)^{n}
$$


whenever $i \in \mathbb{N}, \lambda^{-i} \tau \leq \lambda t$. Moreover, in view of Lemma 3.9 and Remark 3.10, only the case $d+t=r$ needs to be considered.

The remaining assertion will be proved by contradiction. If it were false for some $m, n, Q \in$ $\mathbb{N}, 0<\delta_{1} \leq 1 / 2,0<\delta_{2} \leq 1 / 2,0<s_{0}<1$, and $0 \leq M<\infty$, there would exist a sequence $\varepsilon_{i}$ with $\varepsilon_{i} \downarrow 0$ as $i \rightarrow \infty$ and sequences $X_{i}, T_{i}, d_{i}, r_{i}, t_{i} \zeta_{i}, \mu_{i}$, and $\tau_{i}$ with $i \in \mathbb{N}$ showing that $\varepsilon_{i}$ does not satisfy the assertion.

The argument follows the pattern of Lemma 3.11. First, one could assume for some $T \in G(n+m, n)$

$$
T_{i}=T, \quad r_{i}=1
$$

for $i \in \mathbb{N}$ and then noting \# $X_{i} \leq Q$ that $X_{i} \subset \bar{B}_{M}^{n+m}(0)$ and hence, possibly passing to a subsequence, the existence of real numbers $d, t, \tau$, of $\zeta \in \mathbb{R}^{n+m}$, of a nonempty, closed subset $X$ of $\bar{B}_{M}^{n+m}(0)$, see $[6,2.10 .21]$, and of a stationary, integral $n$ varifold $\mu$ in $U:=\bigcup_{x \in X} B_{1}(x)$, see Allard [1, 6.4], such that $\# X \leq Q$, and, as $i \rightarrow \infty$,

$$
\begin{aligned}
& d_{i} \rightarrow d, \quad t_{i} \rightarrow t, \quad \tau_{i} \rightarrow \tau, \quad \zeta_{i} \rightarrow \zeta, \\
& X_{i} \rightarrow X \quad \text { in Hausdorff distance, } \\
& \int \varphi \mathrm{d} \mu_{i} \rightarrow \int \varphi \mathrm{d} \mu \quad \text { for } \varphi \in C_{\mathrm{c}}^{0}\left(\mathbb{R}^{n+m}\right) \text { with spt } \varphi \subset U,
\end{aligned}
$$

and additionally

$$
T_{x} \mu=T \text { for } \mu \text { almost all } x \in U \text {. }
$$

Clearly,

$$
\begin{aligned}
& d \leq M t, \quad d+t=1, \quad t>0, \quad \lambda^{2} \leq \tau / t \leq \lambda, \\
& \# T(X)=1, \quad \zeta \in \bar{B}_{d}^{n+m}(0) \cap T,
\end{aligned}
$$

and one would readily verify

$$
\begin{gathered}
\mu\left(\bigcup_{x \in X}\left\{y \in B_{t}(x+\zeta):|T(y-x)|>s_{0}|y-x|\right\}\right) \leq\left(Q+1-\delta_{2}\right) \omega_{n} t^{n}, \\
\mu\left(\bigcup_{x \in X} \bar{B}_{\tau}(x)\right) \geq\left(Q+\delta_{1}\right) \omega_{n} \tau^{n} .
\end{gathered}
$$

Moreover, Lemma 3.6 would imply with $S_{x}:=\left\{z \in \mathbb{R}^{n+m}: T^{\perp}(z-x)=0\right\}$ for $x \in \mathbb{R}^{n+m}$

$$
\begin{gathered}
\mu\left(\bigcup_{x \in X} B_{\varrho}(x)\right) \geq\left(Q-\delta_{1}\right) \omega_{n} \varrho^{n} \quad \text { for } 0<\varrho \leq 1, \quad \sum_{x \in X} \theta^{n}(\mu, x) \geq Q, \\
\sum_{x \in X} \theta^{n}(\mu, x)\left(\mathcal{H}^{n}\left\llcorner S_{x}\right)(A) \leq \mu(A) \quad \text { for } A \subset U .\right.
\end{gathered}
$$


Therefore if $x \in X, y \in \operatorname{spt} \mu, T^{\perp}(y) \notin T^{\perp}(X), 0<\left|T^{\perp}(y-x)\right|=h<t$, then one would find

$$
\begin{aligned}
\{z & \left.\in S_{y}:|T(z-x)| \leq s_{0}|z-x|\right\}=S_{y} \cap \bar{B}_{\left(s_{0}^{-2}-1\right)^{-1 / 2} h}\left(x+T^{\perp}(y-x)\right), \\
& \left(\left(1-(h / t)^{2}\right)^{n / 2}-\left(s_{0}^{-2}-1\right)^{-n / 2}(h / t)^{n}\right) \omega_{n} t^{n} \\
\quad & \left(\mathcal{H}^{n}\left\llcorner S_{y}\right)\left(B_{t}(x+\zeta)\right)-\left(\mathcal{H}^{n}\left\llcorner S_{y}\right)\left(\left\{z \in \mathbb{R}^{n+m}:|T(z-x)| \leq s_{0}|z-x|\right\}\right)\right.\right. \\
& \leq\left(\mathcal{H}^{n}\left\llcorner S_{y}\right)\left(\left\{z \in B_{t}(x+\zeta):|T(z-x)|>s_{0}|z-x|\right\}\right)\right. \\
& \leq\left(1-\delta_{2}\right) \omega_{n} t^{n},
\end{aligned}
$$

hence $h \geq \lambda t$, in particular, since $\lambda t \geq \tau$ and $\# T(X)=1$,

$$
(\operatorname{spt} \mu) \cap \bigcup_{x \in X} B_{\tau}(x)=\bigcup_{x \in X} S_{x} \cap B_{\tau}(x), \quad \mu\left(\bigcup_{x \in X} \bar{B}_{\tau}(x)\right)=Q \omega_{n} \tau^{n}
$$

contradicting the previously derived lower bound because $\tau>0$.

Proof of (2) On may first assume $\max \left\{\delta_{1}, \delta_{2}\right\} \leq 1 / 2$, then

$$
\lambda^{2} / 2 \leq \operatorname{dist}(\xi, X) / t \leq \lambda / 2
$$

by part (1), and $1 \leq r / t \leq M+1$ by Lemma 3.9 and Remark 3.10.

The remaining assertion will be proved by contradiction. If it were false for some $m, n, Q \in$ $\mathbb{N}, 0<\delta_{1} \leq 1 / 2,0<\delta_{2} \leq 1 / 2,0 \leq s_{0}<1,0 \leq s<1$, and $0 \leq M<\infty$, there would exist a sequence $\varepsilon_{i}$ with $\varepsilon_{i} \downarrow 0$ as $i \rightarrow \infty$ and sequences $X_{i}, T_{i}, d_{i}, r_{i}, t_{i} \zeta_{i}, \mu_{i}$, and $\xi_{i}$ with $i \in \mathbb{N}$ showing that $\varepsilon_{i}$ does not satisfy the assertion.

The argument follows the pattern of part (1). First, one could assume for some $T \in$ $G(n+m, n)$

$$
T_{i}=T, \quad r_{i}=1
$$

for $i \in \mathbb{N}$ and then noting \# $X_{i} \leq Q$ that $X_{i} \subset \bar{B}_{M}^{n+m}(0)$ and hence, possibly passing to a subsequence, the existence of real numbers $d$, $t$, of $\zeta, \xi \in \mathbb{R}^{n+m}$, of a nonempty, closed subset $X$ of $\bar{B}_{M}^{n+m}(0)$, see $[6,2.10 .21]$, and of a stationary, integral $n$ varifold $\mu$ in $U:=\bigcup_{x \in X} B_{1}(x)$, see Allard [1,6.4], such that $\# X \leq Q$, and, as $i \rightarrow \infty$,

$$
\begin{aligned}
& d_{i} \rightarrow d, \quad t_{i} \rightarrow t, \quad \zeta_{i} \rightarrow \zeta, \quad \xi_{i} \rightarrow \xi, \\
& X_{i} \rightarrow X \quad \text { in Hausdorff distance, } \\
& \int \varphi \mathrm{d} \mu_{i} \rightarrow \int \varphi \mathrm{d} \mu \text { for } \varphi \in C_{\mathrm{c}}^{0}\left(\mathbb{R}^{n+m}\right) \text { with spt } \varphi \subset U,
\end{aligned}
$$

and additionally

$$
T_{x} \mu=T \text { for } \mu \text { almost all } x \in U \text {. }
$$

Clearly,

$$
\begin{aligned}
& d \leq M t, \quad d+t \leq 1, \quad 0<t \leq 1, \\
& \# T(X)=1, \quad \zeta \in \bar{B}_{d}^{n+m}(0) \cap T, \quad \xi \in \operatorname{spt} \mu,
\end{aligned}
$$

and one would readily verify

$$
\mu\left(\bigcup_{x \in X}\left\{y \in B_{t}(x+\zeta):|T(y-x)|>s_{0}|y-x|\right\}\right) \leq\left(Q+1-\delta_{2}\right) \omega_{n} t^{n}
$$


It would hold

$$
\begin{gathered}
0<\operatorname{dist}(\xi, X) / t \leq \lambda / 2, \\
|T(\xi-x)| \leq s|\xi-x| \text { for } x \in X, \quad T^{\perp}(\xi) \notin T^{\perp}(X),
\end{gathered}
$$

hence there would exist $x \in X$ with $|\xi-x| \leq \lambda t / 2$ implying $0<\left|T^{\perp}(\xi-x)\right|<t$. Finally, one would obtain as in the last paragraph of the proof of part (1) with $y$ replaced by $\xi$ that

$$
\lambda t \leq\left|T^{\perp}(\xi-x)\right|
$$

which is incompatible with

$$
\left|T^{\perp}(\xi-x)\right| \leq|\xi-x| \leq \lambda t / 2
$$

because $\lambda t>0$.

3.13 The following fact from multilinear algebra, see $[6,1.3 .1]$ for the notation, will be useful. If $m, n \in \mathbb{N}$, and $S, T \in G(n+m, n)$, then

$$
1-\left\|\Lambda_{n}(T \mid S)\right\|^{2} \leq n\|T-S\|^{2}
$$

in fact, verifying $\left\|\Lambda_{n} f-\Lambda_{n} g\right\| \leq n\|f-g\| \sup \{\|f\|,\|g\|\}^{n-1}$ whenever $f, g \in$ $\operatorname{Hom}\left(\mathbb{R}^{n}, \mathbb{R}^{n}\right)$ and $n>1$, the assertion follows from

$$
\begin{gathered}
1=\left\|\Lambda_{n} \mathbb{1}_{S}\right\|, \quad\left\|\Lambda_{n}(T \mid S)\right\|^{2}=\left\|\Lambda_{n}\left((T \mid S)^{*} \circ(T \mid S)\right)\right\|, \\
\mathbb{1}_{S}-(T \mid S)^{*} \circ T\left|S=\mathbb{1}_{S}-(S \circ T) \circ(T \mid S)=\left(S \circ T^{\perp}\right) \circ\left(T^{\perp} \circ S\right)\right| S, \\
\left\|\mathbb{1}_{S}-(T \mid S)^{*} \circ(T \mid S)\right\| \leq\left\|S \circ T^{\perp}\right\|\left\|T^{\perp} \circ S\right\| \leq\|T-S\|^{2} .
\end{gathered}
$$

3.14 In studying approximations of integral varifolds the following notation will be convenient. Suppose $m, n \in \mathbb{N}$, and $T \in G(n+m, n)$. Then there exist orthogonal projections $\pi: \mathbb{R}^{n+m} \rightarrow \mathbb{R}^{n}, \sigma: \mathbb{R}^{n+m} \rightarrow \mathbb{R}^{m}$ such that $T=\mathrm{im} \pi^{*}$ and $\pi \circ \sigma^{*}=0$, hence

$$
T=\pi^{*} \circ \pi, \quad T^{\perp}=\sigma^{*} \circ \sigma, \quad \mathbb{1}_{\mathbb{R}^{n+m}}=\pi^{*} \circ \pi+\sigma^{*} \circ \sigma .
$$

Whenever $a \in \mathbb{R}^{n+m}, 0<r<\infty, 0<h \leq \infty$ the closed cylinder $C(T, a, r, h)$ is defined by

$$
\begin{aligned}
C(T, a, r, h) & =\left\{x \in \mathbb{R}^{n+m}:|T(x-a)| \leq r \text { and }\left|T^{\perp}(x-a)\right| \leq h\right\} \\
& =\left\{x \in \mathbb{R}^{n+m}:|\pi(x-a)| \leq r \text { and }|\sigma(x-a)| \leq h\right\} .
\end{aligned}
$$

This definition extends Allard's definition in $[1,8.10]$ where $h=\infty$.

Lemma 3.15 (Approximation by $Q$ valued functions) Suppose $m, n, Q \in \mathbb{N}, 0<L<\infty$, $1 \leq M<\infty$, and $0<\delta_{i} \leq 1$ for $i \in\{1,2,3,4,5\}$ with $\delta_{5} \leq\left(2 \gamma_{n} n\right)^{-n} / \omega_{n}$.

Then there exists a positive, finite number $\varepsilon$ with the following property.

If $a, r, h, T, \pi$, and $\sigma$ are as in $3.14, h>2 \delta_{4} r$,

$$
U=\left\{x \in \mathbb{R}^{n+m}: \operatorname{dist}(x, C(T, a, r, h))<2 r\right\},
$$

$\mu$ is an integral $n$ varifold in $U$ with locally bounded first variation,

$$
\begin{gathered}
\left(Q-1+\delta_{1}\right) \omega_{n} r^{n} \leq \mu(C(T, a, r, h)) \leq\left(Q+1-\delta_{2}\right) \omega_{n} r^{n}, \\
\mu\left(C\left(T, a, r, h+\delta_{4} r\right) \sim C\left(T, a, r, h-2 \delta_{4} r\right)\right) \leq\left(1-\delta_{3}\right) \omega_{n} r^{n}, \\
\mu(U) \leq M \omega_{n} r^{n},
\end{gathered}
$$


$0<\varepsilon_{1} \leq \varepsilon, B$ denotes the set of all $x \in C(T, a, r, h)$ with $\theta^{* n}(\mu, x)>0$ such that either $\|\delta \mu\|\left(\bar{B}_{\varrho}(x)\right)>\varepsilon_{1} \mu\left(\bar{B}_{\varrho}(x)\right)^{1-1 / n}$ for some $0<\varrho<2 r$, or $\int_{\bar{B}_{\varrho}(x)}\left|T_{\xi} \mu-T\right| \mathrm{d} \mu(\xi)>\varepsilon_{1} \mu\left(\bar{B}_{\varrho}(x)\right)$ for some $0<\varrho<2 r$,

and $H$ denotes the set of all $x \in C(T, a, r, h)$ such that

$$
\begin{gathered}
\|\delta \mu\|\left(B_{2 r}(x)\right) \leq \varepsilon \mu\left(B_{2 r}(x)\right)^{1-1 / n}, \int_{B_{2 r}(x)}\left|T_{\xi} \mu-T\right| \mathrm{d} \mu(\xi) \leq \varepsilon \mu\left(B_{2 r}(x)\right), \\
\mu\left(\bar{B}_{\varrho}(x)\right) \geq \delta_{5} \omega_{n} \varrho^{n} \text { for } 0<\varrho<2 r,
\end{gathered}
$$

then there exist an $\mathcal{L}^{n}$ measurable subset $Y$ of $\mathbb{R}^{n}$ and a function $f: Y \rightarrow Q_{Q}\left(\mathbb{R}^{m}\right)$ with the following seven properties:

(1) $Y \subset \bar{B}_{r}(\pi(a))$ and $f$ is Lipschitzian with Lip $f \leq L$.

(2) Defining $A=C(T, a, r, h) \sim B$ and $A(y)=\{x \in A: \pi(x)=y\}$ for $y \in \mathbb{R}^{n}$, the sets $A$ and $B$ are Borel sets and there holds

$$
\begin{aligned}
& \sigma(A \cap \operatorname{spt} \mu) \subset \bar{B}_{h-\delta_{4} r}(\sigma(a)), \quad \text { spt } f(y) \subset \sigma(A(y)), \\
& \|f(y)\|=\sigma\left(\theta^{n}(\mu, \cdot) \mathcal{H}^{0}\llcorner A(y))\right.
\end{aligned}
$$

whenever $y \in Y$.

(3) Defining the sets

$$
C=\bar{B}_{r}(\pi(a)) \sim(Y \sim \pi(B)), \quad D=C(T, a, r, h) \cap \pi^{-1}(C),
$$

there holds

$$
\mathcal{L}^{n}(C)+\mu(D) \leq \Gamma_{(3)} \mu(B) .
$$

with $\Gamma_{(3)}=\max \left\{3+2 Q+(12 Q+6) 5^{n}, 4(Q+2) / \delta_{1}\right\}$.

(4) If $x_{1} \in H$, then

$$
\left|\sigma\left(x_{1}-a\right)\right| \leq h-\delta_{4} r
$$

and for $y \in Y \cap \bar{B}_{\lambda_{(4)} r}\left(\pi\left(x_{1}\right)\right)$ there exists $x_{2} \in A(y)$ with $\theta^{n}\left(\mu, x_{2}\right) \in \mathbb{N}$ and

$$
\left|T^{\perp}\left(x_{2}-x_{1}\right)\right| \leq L\left|T\left(x_{2}-x_{1}\right)\right|,
$$

where $0<\lambda_{(4)}<1$ depends only on $n, \delta_{2}$, and $\delta_{4}$. Moreover, $A \cap \operatorname{spt} \mu \subset H$ and

$$
(\pi \bowtie \sigma)\left(H \cap \pi^{-1}(Y)\right)=\operatorname{graph}_{Q} f .
$$

(5) The set $\bar{Y} \sim Y$ has measure 0 with respect to $\mathcal{L}^{n}$ and $\pi(\mu\llcorner H)$.

(6) If $\mathcal{L}^{n}\left(\bar{B}_{r}(\pi(a)) \sim Y\right) \leq(1 / 2) \omega_{n}\left(\lambda_{(4)} r / 6\right)^{n}, 1 \leq q<\infty, P=\left(\theta^{0}(\|S\|, \cdot) \circ \sigma\right) \mathcal{H}^{n}$ is the $Q$ valued plane associated to $S \in Q_{Q}\left(\mathbb{R}^{m}\right)$ via $\sigma$, and $g: Y \rightarrow \mathbb{R}$ is defined by $g(y)=\mathcal{G}(f(y), S)$ for $y \in Y$, then

$$
\begin{aligned}
& \| \operatorname{dist}(\cdot, \text { spt } P) \|_{L^{q}(\mu\llcorner H)} \\
& \quad \leq(12)^{n+1} Q\left(\|g\|_{L^{q}\left(\mathcal{L}^{n}\llcorner Y)\right.}+\Gamma_{(6)} \mathcal{L}^{n}\left(\bar{B}_{r}(\pi(a)) \sim Y\right)^{1 / q+1 / n}\right),
\end{aligned}
$$

where $\Gamma_{(6)}$ is a positive, finite number depending only on $n$, and

$$
\sup \{\operatorname{dist}(x, \operatorname{spt} P): x \in H\} \leq\|g\|_{L^{\infty}\left(\mathcal{L}^{n}\llcorner Y)\right.}+2\left(\mathcal{L}^{n}\left(\bar{B}_{r}(\pi(a)) \sim Y\right) / \omega_{n}\right)^{1 / n} .
$$


(7) For $\mathcal{L}^{n}$ almost all $y \in Y$ the following is true:

(a) $f$ is approximately strongly affinely approximable at $y$.

(b) Whenever $x \in H$ with $\pi(x)=y$

$$
(\pi \bowtie \sigma)\left(T_{x} \mu\right)=\operatorname{Tan}\left(\operatorname{graph}_{Q} \text { ap } A f(y),(y, \sigma(x))\right)
$$

where $\operatorname{Tan}(S, a)$ denotes the classical tangent cone of $S$ at a in the sense of $[6$, 3.1.21].

(c) $\left\|T_{x} \mu-T\right\| \leq \|$ ap $A f(y) \|$ for $x \in H$ with $\pi(x)=y$.

(d) $\|$ ap $A f(y) \|^{2} \leq Q\left(1+(\operatorname{Lip} f)^{2}\right) \max \left\{\left\|T_{x} \mu-T\right\|^{2}: x \in \pi^{-1}(\{y\}) \cap H\right\}$.

Choice of constants One can assume $3 L \leq \delta_{4}$.

Choose $0<s_{0}<1$ close to 1 such that $2\left(s_{0}^{-2}-1\right)^{1 / 2} \leq \delta_{4}$, define

$$
\lambda=\lambda_{3.12}\left(n, \delta_{2}, s_{0}\right) / 4,
$$

choose $s_{0} \leq s<1$ close to 1 satisfying

$$
\left(s^{-2}-1\right)^{1 / 2} \leq \lambda / 4, \quad Q^{1 / 2}\left(s^{-2}-1\right)^{1 / 2} \leq L,
$$

and define $\varepsilon>0$ so small that

$$
\begin{aligned}
\varepsilon & \leq\left(2 \gamma_{n}\right)^{-1}, \quad Q-1+\delta_{1} / 2 \leq\left(1-n \varepsilon^{2}\right)\left(Q-1+\delta_{1}\right), \\
Q-1 / 2 & \leq\left(1-n \varepsilon^{2}\right)(Q-1 / 4), \quad 1-n \varepsilon^{2} \geq 1 / 2,
\end{aligned}
$$

and not larger than the minimum of the following eight numbers

$$
\begin{aligned}
& \varepsilon_{2.4}\left(m, n, 1-\delta_{3} / 2\right), \quad \varepsilon_{2.11}\left(m, n, 1, M, \delta_{3} / 2, s\right), \\
& \varepsilon_{2.11}\left(m, n, Q+1, M, \delta_{2} / 2, s\right), \quad \varepsilon_{2.11}(m, n, Q, M, 1 / 4, s), \\
& \varepsilon_{2.2}\left(m, n, \min \left\{\delta_{2} / 3, \delta_{3} / 2, \delta_{5}\right\}, s, \max \{M, 2\}\right), \quad \varepsilon_{2.11}\left(m, n, Q, M, \delta_{2} / 3, s\right), \\
& \varepsilon_{2.12}\left(m, n, Q, \delta_{5}, \delta_{2}, s, s_{0}, M\right), \quad \varepsilon_{2.12}\left(m, n, Q, 1, \delta_{2}, s, s_{0}, M\right) .
\end{aligned}
$$

Clearly, $\varepsilon_{1}$ satisfies the same inequalities as $\varepsilon$ and one can assume $a=0$, and $r=1$.

Proof of (1) and (2) Since $\theta^{* n}(\mu, \cdot)$ is a Borel function, one may verify that $A$ and $B$ are Borel sets (cp. [6, 2.9.14]).

First, the following basic properties of $A$ are proved: For $x \in A \cap \operatorname{spt} \mu$

$$
\theta_{*}^{n}(\mu, x) \geq \delta_{3} / 2
$$

$$
\begin{gathered}
\left\{\xi \in \pi^{-1}\left(\bar{B}_{1}^{n}(0)\right):|T(\xi-x)|>s|\xi-x|\right\} \subset \sigma^{-1}\left(\bar{B}_{\min \left\{\lambda / 2, \delta_{4}\right\}}(\sigma(x))\right), \\
\sigma(A \cap \operatorname{spt} \mu) \subset \bar{B}_{h-\delta_{4}}^{m}(0) .
\end{gathered}
$$

The first is implied by Lemma 3.4. The second is a consequence of the fact that for $\xi \in$ $\pi^{-1}\left(\bar{B}_{1}^{n}(0)\right)$ with $|T(\xi-x)|>s|\xi-x|$

$$
|\sigma(\xi)-\sigma(x)|<\left(s^{-2}-1\right)^{1 / 2}|\pi(\xi)-\pi(x)| \leq 2\left(s^{-2}-1\right)^{1 / 2} \leq \min \left\{\lambda / 2, \delta_{4}\right\} .
$$

To prove the third, note that Lemma 3.11 applied with

$$
\begin{aligned}
& Q, \delta, X, d, r, t, \text { and } f \text { replaced by } \\
& 1, \delta_{3} / 2,\{x\}, 1,2,1, \text { and } T^{\perp} \mid\{x\}
\end{aligned}
$$

yields

$$
\mu\left(\pi^{-1}\left(\bar{B}_{1}^{n}(0)\right) \cap \sigma^{-1}\left(\bar{B}_{\delta_{4}}(\sigma(x))\right)\right) \geq\left(1-\delta_{3} / 2\right) \omega_{n},
$$


so that $h-\delta_{4}<|\sigma(x)| \leq h$ would be incompatible with

$$
\mu\left(C\left(T, 0,1, h+\delta_{4}\right) \sim C\left(T, 0,1, h-2 \delta_{4}\right)\right) \leq\left(1-\delta_{3}\right) \omega_{n} .
$$

Next, the following assertion will be shown:

If $X \subset A \cap \operatorname{spt} \mu, \theta^{n}(\mu, x) \in \mathbb{N}_{0}$ for $x \in X$, and $s^{-1}\left|T\left(x_{2}-x_{1}\right)\right| \leq\left|x_{2}-x_{1}\right|$ whenever $x_{1}, x_{2} \in X$, then $\sum_{x \in X} \theta^{n}(\mu, x) \leq Q$.

Using the basic properties of $A$ to verify

$$
\begin{aligned}
\left\{\xi \in B_{1}\left(T^{\perp}(x)\right):|T(\xi-x)|>s|\xi-x|\right\} & \subset \pi^{-1}\left(\bar{B}_{1}^{n}(0)\right) \cap \sigma^{-1}\left(\bar{B}_{\delta_{4}}(\sigma(x))\right) \\
& \subset C(T, 0,1, h)
\end{aligned}
$$

there holds

$$
\begin{aligned}
\mu\left(\bigcup_{x \in X}\left\{\xi \in B_{1}\left(T^{\perp}(x)\right):|T(\xi-x)|>s|\xi-x|\right\}\right) & \leq \mu(C(T, 0,1, h)) \\
& \leq\left(Q+1-\delta_{2}\right) \omega_{n}
\end{aligned}
$$

and Lemma 3.11 applied with

$$
\begin{aligned}
& Q, \delta, d, r, t \text {, and } f \text { replaced by } \\
& Q+1, \delta_{2} / 2,1,2,1 \text {, and } T^{\perp} \mid X
\end{aligned}
$$

yields

$$
\sum_{x \in X} \theta^{n}(\mu, x)<Q+\delta_{2} / 2
$$

hence $\sum_{x \in X} \theta^{n}(\mu, x) \leq Q$ and the assertion is proved. It implies, in particular, $\sum_{x \in A(y)} \theta^{n}(\mu, x) \leq Q$ whenever $y \in \bar{B}_{1}^{n}(0)$ and $\theta^{n}(\mu, x) \in \mathbb{N}_{0}$ for each $x \in A(y)$.

Let $Y$ be the set of all $y \in \bar{B}_{1}^{n}(0)$ such that

$$
\sum_{x \in A(y)} \theta^{n}(\mu, x)=Q \text { and } \theta^{n}(\mu, x) \in \mathbb{N}_{0} \text { for } x \in A(y),
$$

$Z$ be the set of all $z \in \bar{B}_{1}^{n}(0)$ such that

$$
\sum_{x \in A(z)} \theta^{n}(\mu, x) \leq Q-1 \text { and } \theta^{n}(\mu, x) \in \mathbb{N}_{0} \text { for } x \in A(z),
$$

and $N=\bar{B}_{1}^{n}(0) \sim(Y \cup Z)$. Clearly, $Y \cap Z=\emptyset$. Note by the concluding remark of the preceding paragraph $\mathcal{L}^{n}(N)=0$ because $\theta^{n}(\mu, x) \in \mathbb{N}_{0}$ for $\mathcal{H}^{n}$ almost all $x \in U$. Since $\theta^{n}(\mu, \cdot)$ is a Borel function whose domain is a Borel set and $A$ is a Borel set, $Y$ and $Z$ are $\mathcal{L}^{n}$ measurable by $[6,3.2 .22(3)]$. Let $f: Y \rightarrow Q_{Q}\left(\mathbb{R}^{m}\right)$ be defined by

$$
f(y)=\sigma_{\#}\left(\sum_{x \in A(y)} \theta^{n}(\mu, x) \llbracket x \rrbracket\right) \text { whenever } y \in Y .
$$

One infers from the assertion of the preceding paragraph and 2.14

$$
\mathcal{G}\left(f\left(y_{2}\right), f\left(y_{1}\right)\right) \leq Q^{1 / 2}\left(s^{-2}-1\right)^{1 / 2}\left|y_{2}-y_{1}\right| \text { for } y_{1}, y_{2} \in Y .
$$

(1) and (2) are now evident. 
Proof of (3) For the estimate some preparations are needed. Let $v$ denote the Radon measure defined by the requirement

$$
v(X)=\int_{X} J^{\mu} T \mathrm{~d} \mu \text { for every Borel subset } X \text { of } U
$$

where $J^{\mu}$ denotes the Jacobian with respect $\mu$. Note by $[6,2.9 .8]$

$$
\left|T_{x} \mu-T\right| \leq \varepsilon \text { for } \mu \text { almost all } x \in A,
$$

hence $1-J^{\mu} T(x) \leq 1-\left(J^{\mu} T\right)(x)^{2} \leq n \varepsilon^{2}$ by 3.13 . Therefore

$$
\left(1-n \varepsilon^{2}\right) \mu\llcorner A \leq v\llcorner A .
$$

This implies the coarea estimate

$$
\begin{aligned}
& \left(1-n \varepsilon^{2}\right) \mu\left(C(T, 0,1, h) \cap \pi^{-1}(W)\right) \\
& \quad \leq \mu\left(B \cap \pi^{-1}(W)\right)+Q \mathcal{L}^{n}(Y \cap W)+(Q-1) \mathcal{L}^{n}(Z \cap W)
\end{aligned}
$$

for every subset $W$ of $\mathbb{R}^{n}$; in fact the estimate holds true for every Borel set by [6, 3.2.22(3)] and $\pi\left(\mu\llcorner B)\right.$ is a Radon measure by $[6,2.2 .17]$. Also note that in view of the choice of $\Gamma_{(3)}$ one can assume

$$
\mu(B) \leq\left(\delta_{1} / 4\right) \omega_{n},
$$

which implies $\mathcal{L}^{n}(Y)>0$ because it follows from the coarea estimate applied with $W=$ $\bar{B}_{1}^{n}(0)$

$$
\begin{aligned}
\left(Q-1+\delta_{1} / 2\right) \omega_{n} & \leq\left(1-n \varepsilon^{2}\right) \mu(C(T, 0,1, h)) \\
& \leq \mu(B)+Q \mathcal{L}^{n}(Y)+(Q-1) \mathcal{L}^{n}(Z) \\
& \leq\left(\delta_{1} / 4\right) \omega_{n}+\left(Q-1+\delta_{1} / 4\right) \omega_{n}+\mathcal{L}^{n}(Y)-\left(\delta_{1} / 4\right) \mathcal{L}^{n}(Z),
\end{aligned}
$$

hence $\mathcal{L}^{n}(Z) \leq\left(4 / \delta_{1}\right) \mathcal{L}^{n}(Y)$.

In order to derive an upper bound for the $\mathcal{L}^{n}$ measure of $Z$, the following assertion will be proved.

If $z \in Z$ with $\theta^{n}\left(\mathcal{L}^{n}\left\llcorner\mathbb{R}^{n} \sim Z, z\right)=0\right.$, then there exist $\zeta \in \mathbb{R}^{n}$ and $0<t<\infty$ with $z \in \bar{B}_{t}(\zeta) \subset \bar{B}_{1}^{n}(0)$ and $\mathcal{L}^{n}\left(\bar{B}_{5 t}(\zeta)\right) \leq 6 \cdot 5^{n} \mu\left(B \cap \pi^{-1}\left(\bar{B}_{t}(\zeta)\right)\right)$.

Since $\mathcal{L}^{n}(Y)>0$, some element $\bar{B}_{t}(\zeta)$ of the family of balls

$$
\left\{\bar{B}_{\theta}((1-\theta) z): 0<\theta \leq 1\right\}
$$

will satisfy

$$
z \in \bar{B}_{t}(\zeta) \subset \bar{B}_{1}^{n}(0), \quad 0<\mathcal{L}^{n}\left(Y \cap \bar{B}_{t}(\zeta)\right) \leq(1 / 2) \mathcal{L}^{n}\left(Z \cap \bar{B}_{t}(\zeta)\right) .
$$

Hence there exists $y \in Y \cap B_{t}(\zeta)$. Noting for $\xi \in A(y)$ with $\theta^{n}(\mu, \xi)>0$, and $\kappa \in \mathbb{R}^{n+m}$ with $\left|\xi+\pi^{*}(\zeta-y)-\kappa\right|<t$,

$$
\begin{gathered}
t \leq 1, \quad \pi(\xi)=y, \\
|\pi(\kappa)-\zeta|=\left|\pi\left(\xi+\pi^{*}(\zeta-y)-\kappa\right)\right| \leq\left|\xi+\pi^{*}(\zeta-y)-\kappa\right|<t, \\
B_{t}\left(\xi+\pi^{*}(\zeta-y)\right) \subset \pi^{-1}\left(\bar{B}_{t}(\zeta)\right),
\end{gathered}
$$

and, recalling the basic properties of $A$,

$$
\left\{\kappa \in B_{t}\left(\xi+\pi^{*}(\zeta-y)\right):|T(\kappa-\xi)|>s|\kappa-\xi|\right\} \subset C(T, 0,1, h) \cap \pi^{-1}\left(\bar{B}_{t}(\zeta)\right),
$$


one can apply Lemma 3.11 with

$$
\begin{aligned}
& \delta, X, d, r, \text { and } f \text { replaced by } \\
& 1 / 4,\left\{\xi \in A(y): \theta^{n}(\mu, \xi)>0\right\}, t, 2, \text { and } \\
& \eta_{-\pi^{*}(\zeta-y), 1} \mid\left\{\xi \in A(y): \theta^{n}(\mu, \xi)>0\right\}
\end{aligned}
$$

to obtain

$$
(Q-1 / 4) \omega_{n} t^{n} \leq \mu\left(C(T, 0,1, h) \cap \pi^{-1}\left(\bar{B}_{t}(\zeta)\right)\right) .
$$

The coarea estimate with $W=\bar{B}_{t}(\zeta)$ now implies

$$
\begin{aligned}
& (Q-1 / 2) \omega_{n} t^{n} \\
& \quad \leq \mu\left(B \cap \pi^{-1}\left(\bar{B}_{t}(\zeta)\right)\right)+Q \mathcal{L}^{n}\left(Y \cap \bar{B}_{t}(\zeta)\right)+(Q-1) \mathcal{L}^{n}\left(Z \cap \bar{B}_{t}(\zeta)\right) \\
& =\mu\left(B \cap \pi^{-1}\left(\bar{B}_{t}(\zeta)\right)\right)+(Q-1 / 2) \omega_{n} t^{n} \\
& \quad+(1 / 2) \mathcal{L}^{n}\left(Y \cap \bar{B}_{t}(\zeta)\right)-(1 / 2) \mathcal{L}^{n}\left(Z \cap \bar{B}_{t}(\zeta)\right),
\end{aligned}
$$

hence

$$
(2 / 3) \mathcal{L}^{n}\left(\bar{B}_{t}(\zeta)\right) \leq \mathcal{L}^{n}\left(Z \cap \bar{B}_{t}(\zeta)\right) \leq 4 \mu\left(B \cap \pi^{-1}\left(\bar{B}_{t}(\zeta)\right)\right)
$$

and the assertion follows.

$\mathcal{L}^{n}$ almost all $z \in Z$ satisfy the assumptions of the assertion of the preceding paragraph (cf. [6, 2.9.11]) and Vitali's covering theorem (cf. [6, 2.8.5]) implies

$$
\mathcal{L}^{n}(Z) \leq 6 \cdot 5^{n} \mu(B) .
$$

Clearly,

$$
\mathcal{L}^{n}(\pi(B)) \leq \mathcal{H}^{n}(B) \leq \mu(B) .
$$

Since $C \sim N \subset Z \cup \pi(B)$, it follows

$$
\mathcal{L}^{n}(C) \leq\left(1+6 \cdot 5^{n}\right) \mu(B) .
$$

Finally, applying the coarea estimate with $W=C$ yields

$$
\left(1-n \varepsilon^{2}\right) \mu(D) \leq \mu(B)+Q \mathcal{L}^{n}(C) \leq\left(1+Q+6 Q \cdot 5^{n}\right) \mu(B) .
$$

Proof of (4) Assuming now that $x_{1}$ and $y$ satisfy the conditions of (4), it will be shown that one can take $\lambda_{(4)}=\lambda$. Verifying

$$
\left\{\xi \in \pi^{-1}\left(\bar{B}_{1}^{n}(0)\right):\left|T\left(\xi-x_{1}\right)\right|>s\left|\xi-x_{1}\right|\right\} \subset \sigma^{-1}\left(\bar{B}_{\min \left\{\lambda / 2, \delta_{4}\right\}}\left(\sigma\left(x_{1}\right)\right)\right),
$$

defining $\delta_{6}=\min \left\{\delta_{2} / 3, \delta_{3} / 2\right\}$ and applying Lemma 3.2 with

$$
\begin{aligned}
& \delta, M, a, r, d, t, \text { and } \zeta \text { replaced by } \\
& \min \left\{\delta_{5}, \delta_{6}\right\}, \max \{M, 2\}, x_{1}, 2,1,1, \text { and }-T\left(x_{1}\right)
\end{aligned}
$$

yields the lower bound

$$
\mu\left(\pi^{-1}\left(\bar{B}_{1}^{n}(0)\right) \cap \sigma^{-1}\left(\bar{B}_{\min \left\{\lambda / 2, \delta_{4}\right\}}\left(\sigma\left(x_{1}\right)\right)\right)\right) \geq\left(1-\delta_{6}\right) \omega_{n}
$$


so that $h-\delta_{4}<\left|\sigma\left(x_{1}\right)\right| \leq h$ would be incompatible with

$$
\mu\left(C\left(T, 0,1, h+\delta_{4}\right) \sim C\left(T, 0,1, h-2 \delta_{4}\right)\right) \leq\left(1-\delta_{3}\right) \omega_{n}
$$

and the first part of (4) follows.

To prove the second part, one defines $X=\left\{\xi \in A(y): \theta^{n}(\mu, \xi) \in \mathbb{N}\right\}$ and first observes that Lemma 3.11 applied with

$$
\begin{aligned}
& \delta, d, r, t, \text { and } f \text { replaced by, } \\
& \delta_{2} / 3,1,2,1 \text {, and } \eta_{\pi^{*}(y), 1} \mid X
\end{aligned}
$$

yields

$$
\mu\left(\bigcup_{x \in X}\left\{\xi \in B_{1}\left(x-\pi^{*}(y)\right):|T(\xi-x)|>s|\xi-x|\right\}\right) \geq\left(Q-\delta_{2} / 3\right) \omega_{n}
$$

On the other hand

$$
\mu(C(T, 0,1, h)) \leq\left(Q+1-\delta_{2}\right) \omega_{n} .
$$

Therefore, using the basic properties of $A$ and the lower bound derived in the previous paragraph, for some $x \in X$

$$
C(T, 0,1, h) \cap \sigma^{-1}\left(\bar{B}_{\lambda / 2}\left(\sigma\left(x_{1}\right)\right)\right) \cap \sigma^{-1}\left(\bar{B}_{\lambda / 2}(\sigma(x))\right) \neq \emptyset,
$$

hence $\left|\sigma\left(x_{1}-x\right)\right| \leq \lambda$ and

$$
\operatorname{dist}\left(x_{1}, X\right) \leq\left|\pi\left(x_{1}-x\right)\right|+\left|\sigma\left(x_{1}-x\right)\right| \leq 2 \lambda=\lambda_{3.12}\left(n, \delta_{2}, s_{0}\right) / 2 \leq 1 .
$$

Now, the point $x_{2} \in X$ may be constructed by applying Lemma 3.12(2) with

$$
\begin{aligned}
& \delta_{1}, \lambda, d, r, t, \zeta, \text { and } \xi \text { replaced by } \\
& \delta_{5}, \lambda_{3.12}\left(n, \delta_{2}, s_{0}\right), 1,2,1,-\pi^{*}(y), \text { and } x_{1}
\end{aligned}
$$

noting

$$
\left\{\xi \in B_{1}\left(x-\pi^{*}(y)\right):|T(\xi-x)|>s_{0}|\xi-x|\right\} \subset C(T, 0,1, h)
$$

for $x \in X$.

Since $\varepsilon_{1} \leq \varepsilon \leq\left(2 \gamma_{n}\right)^{-1}$ and $\delta_{5} \omega_{n} \leq\left(2 \gamma_{n} n\right)^{-n}$, the inclusion $A \cap$ spt $\mu \subset H$ follows from [11,2.5]. Clearly, $(\pi \bowtie \sigma)\left(A \cap \operatorname{spt} \mu \cap \pi^{-1}(Y)\right)=\operatorname{graph}_{Q} f$ by (2). Taking $y=\pi\left(x_{1}\right)$, one obtains $H \cap \pi^{-1}(Y) \subset A \cap \operatorname{spt} \mu$, hence $(\pi \bowtie \sigma)\left(H \cap \pi^{-1}(Y)\right)=\operatorname{graph}_{Q} f$ by the preceding inclusion.

Proof of (5) Recalling $\left(\mu\llcorner A) / 2 \leq v\left\llcorner A\right.\right.$ and $\mathcal{L}^{n}(N)=0$, it is enough to prove

$$
\bar{Y} \subset N \cup Y, \quad \pi^{-1}(\bar{Y}) \cap H \subset A \cap \operatorname{spt} \mu
$$

in view of the coarea formula [6, 3.2.22(3)].

Suppose for this purpose $y \in \bar{Y}$. Since $f$ is Lipschitzian, there exists a unique $S \in Q_{Q}\left(\mathbb{R}^{m}\right)$ such that

$$
(y, S) \in \overline{\operatorname{graph} f} .
$$

Note $\{y\} \times$ spt $S=\left(\{y\} \times \mathbb{R}^{m}\right) \cap \overline{\operatorname{graph}_{Q} f}$ and define $R=\pi^{-1}(\{y\}) \cap \sigma^{-1}$ (spt $\left.S\right)$. Since $A \cap \operatorname{spt} \mu$ is closed (cp. [6, 2.9.14]),

$$
R \subset A \cap \operatorname{spt} \mu
$$


and (4) implies $H \cap \pi^{-1}(\{y\}) \subset R$, the second inclusion follows.

Choose a sequence $y_{i} \in Y$ with $y_{i} \rightarrow y$ as $i \rightarrow \infty$ and abbreviate

$$
X_{i}=\left\{\xi \in A\left(y_{i}\right): \theta^{n}(\mu, \xi) \in \mathbb{N}\right\} \text { for } i \in \mathbb{N} .
$$

Now, Lemma 3.11 applied with

$$
\delta, X, d, r \text {, and } f \text { replaced by }
$$$$
1 / 4, X_{i}, 0,2 \text {, and } \mathbb{1}_{X_{i}}
$$

yields for $i \in \mathbb{N}$

$$
\mu\left(\bigcup_{x \in X_{i}} \bar{B}_{t}(x)\right) \geq(Q-1 / 4) \omega_{n} t^{n} \quad \text { whenever } 0<t<2 .
$$

Since spt $f\left(y_{i}\right) \rightarrow \operatorname{spt} S$ in Hausdorff distance as $i \rightarrow \infty$ the same estimate holds with $X_{i}$ replaced by $R$ and

$$
Q-1 / 4 \leq \limsup _{t \downarrow 0} \frac{\mu\left(\bigcup_{x \in R} \bar{B}_{t}(x)\right)}{\omega_{n} t^{n}} \leq \sum_{x \in R} \theta^{* n}(\mu, x)
$$

implies $y \notin Z$, hence the first inclusion.

Proof of (6) Let $\psi:=\mu\left\llcorner H\right.$. Using $(\pi(\psi))\left\llcorner Y \leq 2\left(\pi(v\llcorner H))\left\llcorner Y \leq 2 Q \mathcal{L}^{n}\llcorner Y\right.\right.\right.$ and

$$
\left\{x \in H \cap \pi^{-1}(Y): \operatorname{dist}(x, \operatorname{spt} P)>\gamma\right\} \subset H \cap \pi^{-1}(\{y \in Y: g(y)>\gamma\})
$$

for $0<\gamma<\infty$ by (4), one infers

$$
\| \operatorname{dist}(\cdot, \text { spt } P)\left\|_{L^{q}\left(\mu\left\llcorner H \cap \pi^{-1}(Y)\right)\right.} \leq 2 Q\right\| g \|_{L^{q}\left(\mathcal{L}^{n}\llcorner Y)\right.} .
$$

Hence only $\| \operatorname{dist}(\cdot$, spt $P) \|_{L^{q}\left(\mu\left\llcorner H \sim \pi^{-1}(Y)\right)\right.}$ needs to be estimated in the first part of (6).

Since $\lambda=\lambda_{(4)}$, whenever $z \in \bar{B}_{1}^{n}(0) \sim \bar{Y}$ there exist $\zeta \in \mathbb{R}^{n}$ and $0<t \leq \lambda / 6$ such that

$$
z \in \bar{B}_{t}(\zeta) \subset \bar{B}_{1}^{n}(0), \quad \mathcal{L}^{n}\left(\bar{B}_{t}(\zeta) \cap Y\right)=\mathcal{L}^{n}\left(\bar{B}_{t}(\zeta) \sim Y\right)
$$

as may be verified by consideration of the family of closed balls

$$
\left\{\bar{B}_{\theta}((1-\theta) z): 0<\theta \leq \lambda / 6\right\} .
$$

Therefore $[6,2.8 .5]$ yields a countable set $I$ and $\zeta_{i} \in \mathbb{R}^{n}, 0<t_{i} \leq \lambda / 6$ and $y_{i} \in Y \cap \bar{B}_{t_{i}}\left(\zeta_{i}\right)$ for each $i \in I$ such that

$$
\begin{gathered}
\bar{B}_{t_{i}}\left(\zeta_{i}\right) \subset \bar{B}_{1}^{n}(0), \quad \mathcal{L}^{n}\left(\bar{B}_{t_{i}}\left(\zeta_{i}\right) \cap Y\right)=\mathcal{L}^{n}\left(\bar{B}_{t_{i}}\left(\zeta_{i}\right) \sim Y\right), \\
\bar{B}_{t_{i}}\left(\zeta_{i}\right) \cap \bar{B}_{t_{j}}\left(\zeta_{j}\right)=\emptyset \quad \text { whenever } i, j \in I \text { with } i \neq j, \\
\bar{B}_{1}^{n}(0) \sim \bar{Y} \subset \bigcup_{i \in I} E_{i} \subset \bar{B}_{1}^{n}(0)
\end{gathered}
$$

where $E_{i}=\bar{B}_{5 t_{i}}\left(\zeta_{i}\right) \cap \bar{B}_{1}^{n}(0)$ for $i \in I$. Let

$$
h_{i}:=\mathcal{G}\left(f\left(y_{i}\right), S\right), \quad X_{i}:=\left\{\xi \in A\left(y_{i}\right): \theta^{n}(\mu, \xi) \in \mathbb{N}\right\}
$$

for $i \in I, J:=\left\{i \in I: h_{i} \geq 18 t_{i}\right\}$, and $K:=I \sim J$.

In view of (5) there holds

$$
\|d\|_{L^{q}\left(\mu\left\llcorner H \sim \pi^{-1}(Y)\right)\right.} \leq\|d\|_{L^{q}\left(\psi\left\llcorner\pi^{-1}\left(\bigcup_{j \in J} E_{j}\right)\right)\right.}+\|d\|_{L^{q}\left(\psi\left\llcorner\pi^{-1}\left(\bigcup_{i \in K} E_{i}\right)\right)\right.}
$$


for every $\psi$ measurable function $d: \mathbb{R}^{n+m} \rightarrow[0, \infty[$. In order to estimate the terms on the right hand side for $d=\operatorname{dist}(\cdot$, spt $P)$, two observations will be useful:

(i) If $i \in I$ and $x_{1} \in H \cap \pi^{-1}\left(E_{i}\right)$, then $\operatorname{dist}\left(x_{1}\right.$, spt $\left.P\right) \leq 6 t_{i}+h_{i}$.

(ii) There holds $\psi\left(\pi^{-1}\left(E_{i}\right)\right) \leq(Q+1) \omega_{n}\left(12 t_{i}\right)^{n}$ whenever $i \in I$.

Suppose for the proof of the observations that $i \in I$ and $x_{1} \in H \cap \pi^{-1}\left(E_{i}\right)$. Noting $\left|\pi\left(x_{1}\right)-y_{i}\right| \leq 6 t_{i} \leq \lambda$, (4) yields a point $x_{2} \in X_{i}$ and

$$
\left|T^{\perp}\left(x_{2}-x_{1}\right)\right| \leq L\left|T\left(x_{2}-x_{1}\right)\right|=L\left|\pi\left(x_{1}\right)-y_{i}\right| \leq 6 t_{i},
$$

implying

$$
\operatorname{dist}\left(x_{1}, \operatorname{spt} P\right) \leq\left|T^{\perp}\left(x_{2}-x_{1}\right)\right|+\operatorname{dist}\left(x_{2}, \operatorname{spt} P\right) \leq 6 t_{i}+h_{i},
$$

hence (i). Moreover,

$$
\left|x_{2}-x_{1}\right| \leq\left|T\left(x_{2}-x_{1}\right)\right|+\left|T^{\perp}\left(x_{2}-x_{1}\right)\right| \leq 12 t_{i}, \quad x_{1} \in \bar{B}_{12 t_{i}}\left(x_{2}\right),
$$

hence

$$
H \cap \pi^{-1}\left(E_{i}\right) \subset \bigcup_{x \in X_{i}} \bar{B}_{12 t_{i}}(x)
$$

Therefore noting

$$
\left\{y \in B_{1}\left(x-\pi^{*}\left(y_{i}\right)\right):|T(y-x)|>s_{0}|y-x|\right\} \subset C(T, 0,1, h)
$$

for $x \in X_{i}$ by (2) and the choice $s_{0}$, Lemma 3.12(1) applied with

$$
\begin{aligned}
& \delta_{1}, s, \lambda, X, d, r, t, \zeta, \text { and } \tau \text { replaced by } \\
& 1,0, \lambda_{3.12}\left(n, \delta_{2}, s_{0}\right), X_{i}, 1,2,1,-\pi^{*}\left(y_{i}\right), \text { and } 12 t_{i}
\end{aligned}
$$

yields (ii) and the observations are proved.

Now, the first term will be estimated. Note, if $j \in J$, then by the first observation (i)

$$
\begin{aligned}
& \operatorname{dist}(x, \text { spt } P) \leq(4 / 3) h_{j} \quad \text { whenever } x \in H \cap \pi^{-1}\left(E_{j}\right), \\
& (4 / 3) h_{j} \leq 2 \mathcal{G}(f(y), S) \quad \text { whenever } y \in Y \cap \bar{B}_{t_{j}}\left(\zeta_{j}\right),
\end{aligned}
$$

because

$$
\mathcal{G}(f(y), S) \geq \mathcal{G}\left(f\left(y_{j}\right), S\right)-L\left|y-y_{j}\right| \geq h_{j}-2 L t_{j} \geq(2 / 3) h_{j} .
$$

Using this fact and the preceding observations (i) and (ii), one estimates with $J(\gamma):=\{j \in$ $\left.J:(4 / 3) h_{j}>\gamma\right\}$ for $0<\gamma<\infty$

$$
\begin{aligned}
& \psi\left(\pi^{-1}\left(\bigcup_{j \in J} E_{j}\right) \cap\left\{x \in \mathbb{R}^{n+m}: \operatorname{dist}(x, \text { spt } P)>\gamma\right\}\right) \leq \sum_{j \in J(\gamma)} \psi\left(\pi^{-1}\left(E_{j}\right)\right) \\
& \leq \sum_{j \in J(\gamma)}(Q+1) \omega_{n}\left(12 t_{j}\right)^{n} \leq(Q+1)(12)^{n} \mathcal{L}^{n}\left(\bigcup_{j \in J(\gamma)} \bar{B}_{t_{j}}\left(\zeta_{j}\right)\right) \\
& \quad \leq 2(Q+1)(12)^{n} \mathcal{L}^{n}\left(\bigcup_{j \in J(\gamma)} \bar{B}_{t_{j}}\left(\zeta_{j}\right) \cap Y\right) \\
& \quad \leq 2(Q+1)(12)^{n} \mathcal{L}^{n}(\{y \in Y: \mathcal{G}(f(y), S)>\gamma / 2\}),
\end{aligned}
$$


hence

$$
\| \operatorname{dist}(\cdot, \text { spt } P)\left\|_{L^{q}\left(\psi\left\llcorner\pi^{-1}\left(\bigcup_{j \in J} E_{j}\right)\right)\right.} \leq\left(2(Q+1)(12)^{n}\right) 2\right\| g \|_{L^{q}\left(\mathcal{L}^{n}\llcorner Y)\right.} .
$$

To estimate the second term, one notes, if $i \in K, x \in H \cap \pi^{-1}\left(E_{i}\right)$, then

$$
\operatorname{dist}(x, \operatorname{spt} P)<24 t_{i} .
$$

Therefore one estimates with $K(\gamma):=\left\{i \in K: 24 t_{i}>\gamma\right\}$ for $0<\gamma<\infty$ and $u: \mathbb{R}^{n} \rightarrow \mathbb{R}$ defined by $u=\sum_{i \in I} 2 t_{i} \chi_{\bar{B}_{t_{i}}\left(\zeta_{i}\right)}$

$$
\begin{aligned}
& \psi\left(\pi^{-1}\left(\bigcup_{i \in K} E_{i}\right) \cap\left\{x \in \mathbb{R}^{n+m}: \operatorname{dist}(x, \operatorname{spt} P)>\gamma\right\}\right) \leq \sum_{i \in K(\gamma)} \psi\left(\pi^{-1}\left(E_{i}\right)\right) \\
& \leq \sum_{i \in K(\gamma)}(Q+1) \omega_{n}\left(12 t_{i}\right)^{n} \leq(Q+1)(12)^{n} \mathcal{L}^{n}\left(\bigcup_{i \in K(\gamma)} \bar{B}_{t_{i}}\left(\zeta_{i}\right)\right) \\
& \leq(Q+1)(12)^{n} \mathcal{L}^{n}\left(\left\{y \in \mathbb{R}^{n}: u(y)>\gamma /(12)\right\}\right),
\end{aligned}
$$

hence

$$
\| \operatorname{dist}(\cdot \text {, spt } P)\left\|_{L^{q}\left(\psi\left\llcorner\pi^{-1}\left(\bigcup_{i \in K} E_{i}\right)\right)\right.} \leq(Q+1)(12)^{n+1}\right\| u \|_{L^{q}\left(\mathcal{L}^{n}\right)} .
$$

Combining these two estimates, i.e. (III) and (IV), with

$$
\begin{gathered}
\mathcal{L}^{n}\left(\bigcup_{i \in I} \bar{B}_{t_{i}}\left(\zeta_{i}\right)\right) \leq 2 \mathcal{L}^{n}\left(\bar{B}_{1}^{n}(0) \sim Y\right), \\
\int|u|^{q} \mathrm{~d} \mathcal{L}^{n}=\sum_{i \in I}\left(2 t_{i}\right)^{q} \omega_{n}\left(t_{i}\right)^{n} \leq 2^{q} \omega_{n}^{-q / n}\left(\sum_{i \in I} \mathcal{L}^{n}\left(\bar{B}_{t_{i}}\left(\zeta_{i}\right)\right)\right)^{1+q / n}, \\
\|u\|_{L^{q}\left(\mathcal{L}^{n}\right)} \leq 2^{3} \omega_{n}^{-1 / n} \mathcal{L}^{n}\left(\bar{B}_{1}^{n}(0) \sim Y\right)^{1 / q+1 / n},
\end{gathered}
$$

and recalling (I) and (II), one obtains the first part of the conclusion of (6).

To prove the second part, suppose $x_{1} \in H$. Since

$$
\pi\left(x_{1}\right) \in \bar{B}_{\theta}\left((1-\theta) \pi\left(x_{1}\right)\right) \subset \bar{B}_{1}^{n}(0), \quad \mathcal{L}^{n}\left(\bar{B}_{\theta}\left((1-\theta) \pi\left(x_{1}\right)\right) \cap Y\right)>0
$$

for $\left(\mathcal{L}^{n}\left(\bar{B}_{1}^{n}(0) \sim Y\right) / \omega_{n}\right)^{1 / n}<\theta<1$, there exists for any $\delta>0$ a $y \in Y$ with

$$
\begin{aligned}
\mathcal{G}(f(y), S) & \leq\|g\|_{L^{\infty}\left(\mathcal{L}^{n}\llcorner Y)\right.}, \\
\left|\pi\left(x_{1}\right)-y\right| & \leq 2\left(\mathcal{L}^{n}\left(\bar{B}_{1}^{n}(0) \sim Y\right) / \omega_{n}\right)^{1 / n}+\delta,
\end{aligned}
$$

in particular $\left|\pi\left(x_{1}\right)-y\right| \leq \lambda$ for small $\delta$. Therefore (4) may be applied to construct a point $x_{2} \in A(y)$ with $\theta^{n}\left(\mu, x_{2}\right) \in \mathbb{N}$ and

$$
\left|T^{\perp}\left(x_{2}-x_{1}\right)\right| \leq L\left|T\left(x_{2}-x_{1}\right)\right| \leq\left|\pi\left(x_{1}\right)-y\right| .
$$

Finally,

$$
\begin{aligned}
\operatorname{dist}\left(x_{1}, \text { spt } P\right) & \leq \operatorname{dist}\left(x_{2}, \text { spt } P\right)+\left|T^{\perp}\left(x_{2}-x_{1}\right)\right| \\
& \leq \mathcal{G}(f(y), S)+2\left(\mathcal{L}^{n}\left(\bar{B}_{1}^{n}(0) \sim Y\right) / \omega_{n}\right)^{1 / n}+\delta
\end{aligned}
$$

and $\delta$ can be chosen arbitrarily small. 
Proof of (7) Part (7a) follows from (1) and Theorem 2.5. Part (7b) follows from (1), (4) and Theorem 2.5. Parts (7c) and (7d) are consequences of (7b) in conjunction with Allard $[1,8.9(5)]$, noting concerning (7d) that $\left\|D g_{i}(0)\right\| \leq \|$ ap $A f(x) \| \leq \operatorname{Lip} f$ whenever $g_{i}$ : $\mathbb{R}^{n} \rightarrow \mathbb{R}^{m}$ are affine functions such that ap $A f(x)(v)=\sum_{i=1}^{Q} \llbracket g_{i}(v) \rrbracket$ for $v \in \mathbb{R}^{n+m}$ by Almgren [2, 1.1(9)-(11)].

Remark 3.16 The $\mu$ measure of $B$ occuring in (3) can either be estimated by a direct covering argument, as will be done in Corollary 4.8 , or, in order to obtain a slightly more precise estimate, by use of $[11,2.9,2.10]$, as will be done in Theorem 4.11 .

\section{A Sobolev Poincaré type inequality for integral varifolds}

In this section the two main theorems, Theorems 4.4 and 4.11, are proved, the first being a Sobolev Poincaré type inequality at some fixed scale $r$ but involving of necessity mean curvature, the second considering the limit as $r$ tends to 0 . For this purpose the distance of an integral $n$ varifold from a $Q$ valued plane is introduced. One cannot use ordinary planes in Theorem 4.4 (without additional assumptions) as may be seen from the fact that any $Q$ valued plane is stationary with vanishing tilt. In 4.11-4.13 an answer to the Problem posed in the introduction is provided.

Definition 4.1 Suppose $m, n, Q \in \mathbb{N}, 1 \leq q \leq \infty, a \in \mathbb{R}^{n+m}, 0<r<\infty, 0<h \leq$ $\infty, T \in G(n+m, n), P$ is a $Q$ valued plane parallel to $T$ (see Definition 2.13), $\mu$ is an integral $n$ varifold in an open superset of $C(T, a, r, h), A$ is the $\mathcal{H}^{n}$ measurable set of all $x \in T \cap \bar{B}_{r}(T(a))$ such that for some $R(x), S(x) \in Q_{Q}\left(\mathbb{R}^{n+m}\right)$

$$
\begin{aligned}
\|R(x)\| & =\theta^{n}\left(P \llcorner C ( T , a , r , h ) , \cdot ) \mathcal { H } ^ { 0 } \left\llcornerT^{-1}(\{x\}),\right.\right. \\
\|S(x)\| & =\theta^{n}\left(\mu \llcorner C ( T , a , r , h ) , \cdot ) \mathcal { H } ^ { 0 } \left\llcornerT^{-1}(\{x\})\right.\right.
\end{aligned}
$$

and $g: A \rightarrow \mathbb{R}$ is the $\mathcal{H}^{n}$ measurable function defined by $g(x)=\mathcal{G}(R(x), S(x))$ for $x \in A{ }^{1}$

Then the $q$ tilt of $\mu$ with respect to $T$ in $C(T, a, r, h)$ is defined by

$$
T_{q}(\mu, a, r, h, T)=r^{-n / q}\left\|T_{\mu}-T\right\|_{L^{q}(\mu\llcorner C(T, a, r, h))} .
$$

The $q$ height of $\mu$ with respect to $P$ in $C(T, a, r, h)$, denoted by $H_{q}(\mu, a, r, h, P)$, is defined to be the sum of

$$
r^{-1-n / q} \| \operatorname{dist}(\cdot, \text { spt } P) \|_{L^{q}(\mu\llcorner C(T, a, r, h))}
$$

and the infimum of the numbers

$$
r^{-1-n / q}\|g\|_{L^{q}\left(\mathcal{H}^{n}\llcorner Y)\right.}+r^{-1-n / q} \mathcal{H}^{n}\left(T \cap \bar{B}_{r}(T(a)) \sim Y\right)^{1 / q+1 / n}
$$

corresponding to all $\mathcal{H}^{n}$ measurable subsets $Y$ of $A$. Moreover, the $q$ height of $\mu$ in $C(T, a, r, h)$, denoted by $H_{q}(\mu, a, r, h, Q, T)$, is defined to be the infimum of all numbers $H_{q}(\mu, a, r, h, P)$ corresponding to all $Q$ valued planes $P$ parallel to $T$.

Remark 4.2 $T_{q}(\mu, a, r, h, T)$ generalises tiltex ${ }_{\mu}$ in an obvious way.

$H_{q}(\mu, a, r, h, P)$ measures the distance of $\mu$ in $C(T, a, r, h)$ from the $Q$ valued plane $P$. To obtain a reasonable definition of distance, neither the first nor the second summand would be sufficient. The first summand is 0 if $\mu=P\left\llcorner B\right.$ for some $\mathcal{H}^{n}$ measurable set $B$. The




second summand is 0 if $\mu=P+\mathcal{H}^{n}\left\llcorner B\right.$ for some $\mathcal{H}^{n}$ measurable subset $B$ of $C(T, a, r, h)$ with $\mathcal{H}^{n}(B)<\infty$ and $\mathcal{H}^{n}(T(B))=0$. From a more technical point of view, the second summand is added because it is useful in the iteration procedure occurring in Theorem 4.11 where the distance of $Q$ valued planes corresponding to different radii $r$ has to be estimated. The choice of the exponent $1 / q+1 / n$ instead of $1 / q$ for $\mathcal{H}^{n}\left(T \cap \bar{B}_{r}(T(a)) \sim Y\right)$ is motivated by Lemma 3.15(6).

Remark 4.3 One readily checks that $H_{q}(\mu, a, r, h, P)=0$ implies

$$
\mu\llcorner C(T, a, r, h)=P\llcorner C(T, a, r, h)
$$

and $H_{q}(\mu, a, r, h, Q, T)=0, h<\infty$ implies $H_{q}(\mu, a, r, h, P)=0$ for some $Q$ valued plane $P$ parallel to $T$.

More generally, the infima occurring in the definitions of $H_{q}(\mu, a, r, h, P)$ and $H_{q}(\mu, a, r, h, Q, T)$ are attained. However, this latter fact will neither be used nor proved in this work.

Theorem 4.4 Suppose $m, n, Q \in \mathbb{N}, 1 \leq M<\infty$, and $0<\delta \leq 1$.

Then there exists a positive, finite number $\varepsilon$ with the following property.

If $a \in \mathbb{R}^{n+m}, 0<r<\infty, 0<h \leq \infty, T \in G(n+m, n), \delta r<h, \mu$ is an integral $n$ varifold in an open superset of $C(T, a, 3 r, h+2 r)$ with locally bounded first variation satisfying

$$
\begin{gathered}
(Q-1+\delta) \omega_{n} r^{n} \leq \mu(C(T, a, r, h)) \leq(Q+1-\delta) \omega_{n} r^{n}, \\
\mu(C(T, a, r, h+\delta r) \sim C(T, a, r, h-\delta r)) \leq(1-\delta) \omega_{n} r^{n}, \\
\mu(C(T, a, 3 r, h+2 r)) \leq M \omega_{n} r^{n}, \\
\|\delta \mu\|(C(T, a, 3 r, h+2 r)) \leq \varepsilon r^{n-1}, \quad T_{1}(\mu, a, 3 r, h+2 r, T) \leq \varepsilon,
\end{gathered}
$$

$G$ is the set of all $x \in C(T, a, r, h) \cap \operatorname{spt} \mu$ such that

$$
\|\delta \mu\|\left(\bar{B}_{\varrho}(x)\right) \leq\left(2 \gamma_{n}\right)^{-1} \mu\left(\bar{B}_{\varrho}(x)\right)^{1-1 / n} \text { whenever } 0<\varrho<2 r,
$$

and $A$ is the set defined as $G$ with $\varepsilon$ replacing $\left(2 \gamma_{n}\right)^{-1}$, then the following two statements hold:

(1) If $1 \leq q<n, q^{*}=n q /(n-q)$, then

$$
\begin{aligned}
& H_{q^{*}}(\mu\llcorner G, a, r, h, Q, T) \\
& \quad \leq \Gamma_{(1)}\left(T_{q}(\mu, a, 3 r, h+2 r, T)+\left(r^{-n} \mu(C(T, a, r, h) \sim A)\right)^{1 / q}\right)
\end{aligned}
$$

where $\Gamma_{(1)}$ is a positive, finite number depending only on $m, n, Q, M, \delta$, and $q$.

(2) If $n<q \leq \infty$, then

$$
\begin{aligned}
& H_{\infty}(\mu\llcorner G, a, r, h, Q, T) \\
& \quad \leq \Gamma_{(2)}\left(T_{q}(\mu, a, 3 r, h+2 r, T)+\left(r^{-n} \mu(C(T, a, r, h) \sim A)\right)^{1 / q}\right) .
\end{aligned}
$$

where $\Gamma_{(2)}$ is a positive, finite number depending only on $m, n, Q, M, \delta$, and $q$.

Proof Define

$$
\begin{aligned}
\Gamma_{0} & :=\Gamma_{2.12}(m, Q), \quad \Gamma_{1}:=\Gamma_{3.15(3)}(Q, n, \delta / 2), \quad L:=1, \\
\varepsilon_{0} & :=\varepsilon_{3.15}\left(m, n, Q, 1, M, \delta / 2, \delta / 2, \delta / 2, \delta / 2,\left(2 \gamma_{n} n\right)^{-n} / \omega_{n}\right), \quad \varepsilon_{1}:=\varepsilon_{0}, \\
\lambda & :=\lambda_{3.15(4)}(n, \delta / 2, \delta / 2)
\end{aligned}
$$


and choose $0<\varepsilon \leq \varepsilon_{0}$ such that

$$
\begin{gathered}
\varepsilon \leq \varepsilon_{0}\left(n \gamma_{n}\right)^{1-n}, \quad 3^{n} \varepsilon \leq \varepsilon_{0}\left(n \gamma_{n}\right)^{-n}, \\
\Gamma_{1} N(n+m) 3^{n} \varepsilon / \varepsilon_{0} \leq(1 / 2) \omega_{1}(\lambda / 6) \quad \text { if } n=1, \\
\Gamma_{1} N(n+m)\left(3^{n} \varepsilon / \varepsilon_{0}+\left(\varepsilon / \varepsilon_{0}\right)^{n /(n-1)}\right) \leq(1 / 2) \omega_{n}(\lambda / 6)^{n} \quad \text { if } n>1 .
\end{gathered}
$$

Assume $a=0$ and $r=1$. Choose orthogonal projections $\pi: \mathbb{R}^{n+m} \rightarrow \mathbb{R}^{n}, \sigma: \mathbb{R}^{n+m} \rightarrow$ $\mathbb{R}^{m}$ with $\pi \circ \sigma^{*}=0$ and im $\pi^{*}=T$. Applying Lemma 3.15, one obtains sets $Y, B$, and $H$ and a Lipschitzian function $f: Y \rightarrow Q_{Q}\left(\mathbb{R}^{m}\right)$ with the properties listed there. Using Lemma 3.15 (1) (2) and Theorem 2.12 and noting the existence of a retraction of $\mathbb{R}^{m}$ to $\bar{B}_{h}^{m}(0)$ with Lipschitz constant 1 (cf. [6, 4.1.16]), one constructs an extension $g: \bar{B}_{1}^{n}(0) \rightarrow Q_{Q}\left(\mathbb{R}^{m}\right)$ of $f$ with $\operatorname{Lip} g \leq \Gamma_{0}$ and spt $g(x) \subset \bar{B}_{h}^{m}(0)$ for $x \in \bar{B}_{1}^{n}(0)$.

Next, it will be verified that $G \subset H$; in fact for $x \in G$ using [11, 2.5] yields

$$
\begin{aligned}
\mu\left(\bar{B}_{\varrho}(x)\right) & \geq\left(2 \gamma_{n} n\right)^{-n} \varrho^{n} \quad \text { for } 0<\varrho<2, \\
\|\delta \mu\|\left(B_{2}(x)\right) & \leq\|\delta \mu\|(C(T, 0,3, h+2)) \leq \varepsilon \leq \varepsilon_{0} \mu\left(B_{2}(x)\right)^{1-1 / n}, \\
\int_{B_{2}(x)}\left|T_{\xi} \mu-T\right| \mathrm{d} \mu(\xi) & \leq \int_{C(T, 0,3, h+2)}\left|T_{\xi} \mu-T\right| \mathrm{d} \mu(\xi) \leq 3^{n} \varepsilon \leq \varepsilon_{0} \mu\left(B_{2}(x)\right) .
\end{aligned}
$$

In order to be able to apply Lemma 3.15 (6), it will be shown

$$
\mathcal{L}^{n}\left(\bar{B}_{1}^{n}(0) \sim Y\right) \leq(1 / 2) \omega_{n}(\lambda / 6)^{n} .
$$

Let $B_{1}$ be the set of all $x \in B$ such that

$$
\|\delta \mu\|\left(\bar{B}_{\varrho}(x)\right)>\varepsilon_{0} \mu\left(\bar{B}_{\varrho}(x)\right)^{1-1 / n} \text { for some } 0<\varrho<2,
$$

and let $B_{2}$ be the set of all $x \in B$ such that

$$
\int_{\bar{B}_{\varrho}(x)}\left|T_{\xi} \mu-T\right| \mathrm{d} \mu(\xi)>\varepsilon_{0} \mu\left(\bar{B}_{\varrho}(x)\right) \text { for some } 0<\varrho<2 .
$$

Clearly, Besicovitch's covering theorem implies

$$
\mu\left(B_{2}\right) \leq N(n+m)\left(\varepsilon_{0}\right)^{-1} 3^{n} T_{1}(\mu, 0,3, h+2, T) \leq N(n+m) 3^{n} \varepsilon / \varepsilon_{0} .
$$

Moreover, $B_{1}=\emptyset$ if $n=1$, and Besicovitch's covering theorem implies in case $n>1$

$$
\begin{aligned}
\mu\left(B_{1}\right) & \leq N(n+m)\left(\varepsilon_{0}\right)^{n /(1-n)}\|\delta \mu\|(C(T, 0,3, h+2))^{n /(n-1)} \\
& \leq N(n+m)\left(\varepsilon / \varepsilon_{0}\right)^{n /(n-1)} .
\end{aligned}
$$

Therefore the desired estimate is implied by Lemma 3.15(3) and the choice of $\varepsilon$.

To prove part (1), let $1 \leq q<n, q^{*}=n q /(n-q)$, define

$$
\begin{aligned}
& \Gamma_{2}=1+(12)^{n+1} Q \max \left\{1, \Gamma_{3.15(6)}(n)\right\}, \quad \Gamma_{3}=2 \Gamma_{2.10(1)}(m, n, Q, q), \\
& \Gamma_{4}=N(n+m)^{1 / q}\left(\varepsilon_{0}\right)^{-1} 3^{n / q}, \quad \Gamma_{5}=2^{1 / 2} Q m^{1 / 2}, \quad \Gamma_{6}=\Gamma_{0} m^{1 / 2} Q^{1 / 2},
\end{aligned}
$$

choose $S \in Q_{Q}\left(\mathbb{R}^{m}\right)$ such that (see Definition 2.9)

$$
h_{q^{*}}(g, S) \leq \Gamma_{3} t_{q}(g), \quad \text { spt } S \subset \bar{B}_{h}^{m}(0)
$$

with the help of Theorem 2.10(1) noting again [6, 4.1.16] and denote by

$$
P:=\left(\theta^{0}(\|S\|, \cdot) \circ \sigma\right) \mathcal{H}^{n}
$$


the $Q$ valued plane associated to $S$ via $\sigma$. The estimate for $H_{q^{*}}(\mu\llcorner G, 0,1, h, P)$ is obtained by combining the following six inequalities:

$$
\begin{aligned}
H_{q^{*}}(\mu\llcorner G, 0,1, h, P) & \leq \Gamma_{2}\left(h_{q^{*}}(g, S)+\mathcal{L}^{n}\left(\bar{B}_{1}^{n}(0) \sim Y\right)^{1 / q}\right), \\
h_{q^{*}}(g, S) & \leq \Gamma_{3} t_{q}(g), \\
\mathcal{L}^{n}\left(\bar{B}_{1}^{n}(0) \sim Y\right)^{1 / q} & \leq\left(\Gamma_{1}\right)^{1 / q} \mu(B)^{1 / q}, \\
\mu(B \cap A)^{1 / q} & \leq \Gamma_{4} T_{q}(\mu, 0,3, h+2, T), \\
t_{q}(g \mid Y) & \leq \Gamma_{5} T_{q}(\mu, 0,1, h, T), \\
t_{q}\left(g \mid \bar{B}_{1}^{n}(0) \sim Y\right) & \leq \Gamma_{6} \mathcal{L}^{n}\left(\bar{B}_{1}^{n}(0) \sim Y\right)^{1 / q} .
\end{aligned}
$$

The first is implied by Lemma 3.15 (2) (4) (6) and spt $S \subset \bar{B}_{h}^{m}(0)$, the second is implied by the choice of $S$, the third is implied by Lemma 3.15(3), the sixth is elementary (cf. Almgren $[2,1.1(9)-(11)])$. To prove the fourth, note that for every $x \in B \cap A$ there exists $0<\varrho<2$ such that

$$
\varepsilon_{0} \mu\left(\bar{B}_{\varrho}(x)\right)<\int_{\bar{B}_{\varrho}(x)}\left|T_{\xi} \mu-T\right| \mathrm{d} \mu(\xi),
$$

hence by Hölder's inequality

$$
\left(\varepsilon_{0}\right)^{q} \mu\left(\bar{B}_{\varrho}(x)\right)<\int_{\bar{B}_{\varrho}(x)}\left|T_{\xi} \mu-T\right|^{q} \mathrm{~d} \mu(\xi)
$$

and Besicovitch's covering theorem implies the inequality in question. Observing that

$$
\{y \in Y:|\operatorname{ap} A g(y)|>\gamma\} \sim \pi\left(\left\{\xi \in G \cap \pi^{-1}(Y):\left|T_{\xi} \mu-T\right|>\gamma / \Gamma_{5}\right\}\right)
$$

has $\mathcal{L}^{n}$ measure 0 by Lemma 3.15 (7d) and Almgren [2, 1.1(9)-(11)], the fifth inequality is a consequence of

$$
\begin{aligned}
& \mathcal{L}^{n}(\{y \in Y: \mid \text { ap } A g(y) \mid>\gamma\}) \\
& \quad \leq \mathcal{H}^{n}\left(\left\{\xi \in G \cap \pi^{-1}(Y):\left|T_{\xi} \mu-T\right|>\gamma / \Gamma_{5}\right\}\right) \\
& \quad \leq \mu\left(\left\{\xi \in G \cap \pi^{-1}(Y):\left|T_{\xi} \mu-T\right|>\gamma / \Gamma_{5}\right\}\right) .
\end{aligned}
$$

The proof of part (2) exactly parallels the proof of part (1) with $\infty$ and Theorem 2.10(2) replacing $q^{*}$ and Theorem $2.10(1)$.

Remark 4.5 The $\mu$ measure of $C(T, a, r, h) \sim A$ could be estimated using Besicovitch's covering theorem as follows: If $\mu$ satisfies $\left(H_{p}\right)$ with $1 \leq p \leq n, \psi=\|\delta \mu\|$ if $p=1$ and $\psi=\left|\vec{H}_{\mu}\right|^{p} \mu$ if $p>1$, then

$$
\begin{aligned}
& \mu(C(T, a, r, h) \sim A) \\
& \quad \leq N(n+m) \varepsilon^{-n p /(n-p)} \psi(C(T, a, 3 r, h+2 r))^{n /(n-p)} \quad \text { if } p<n, \\
& C(T, a, r, h) \cap(\operatorname{spt} \mu) \sim A=\emptyset \text { if } p=n \text { and } \psi(C(T, a, 3 r, h+2 r)) \leq \varepsilon^{n} ;
\end{aligned}
$$

in fact if $x \in C(T, a, r, h) \cap($ spt $\mu) \sim A$ the definition of $A$ implies for some $0<\varrho<2 r$ by Hölder's inequality

$$
\begin{gathered}
\varepsilon \mu\left(\bar{B}_{\varrho}(x)\right)^{1-1 / n}<\psi\left(\bar{B}_{\varrho}(x)\right)^{1 / p} \mu\left(\bar{B}_{\varrho}(x)\right)^{1-1 / p}, \quad p<n, \\
\mu\left(\bar{B}_{\varrho}(x)\right) \leq \varepsilon^{-n p /(n-p)} \psi\left(\bar{B}_{\varrho}(x)\right)^{n /(n-p)} .
\end{gathered}
$$


Clearly, $A$ and $\varepsilon$ can be replaced by $G$ and $\left(2 \gamma_{n}\right)^{-1}$.

However, this estimate would not be sufficient to prove Theorem 4.11 in the limiting case.

Remark 4.6 The term $\mu\llcorner G$ cannot be replaced by $\mu$ neither in part (1) nor, if $n>1$, in part (2) because otherwise the respective part of Theorem 4.11 would hold with the condition $\alpha q_{2} \leq n p /(n-p)$ replaced by $\alpha q_{1} \leq n p /(n-p)$ in part (1) and $p=n$ replaced by $p>n / 2$ in part (2) which is not the case by [11, 1.2], see Remark 4.13 .

On the other hand one readily infers from the definition of the $q^{*}$ height that

$$
\begin{aligned}
& H_{q^{*}}(\mu, a, r, h, Q, T) \\
& \quad \leq H_{q^{*}}\left(\mu\llcorner G, a, r, h, Q, T)+\left(2 h / r+\omega_{n}^{1 / n}\right)\left(r^{-n} \mu(C(T, a, r, h) \sim G)\right)^{1 / q^{*}} .\right.
\end{aligned}
$$

Remark 4.7 Part (2) can be sharpened using Lorentz spaces to

$$
\begin{aligned}
& H_{\infty}(\mu\llcorner G, a, r, h, Q, T) \\
& \quad \leq \Gamma\left(T_{n, 1}(\mu, a, 3 r, h+2 r, T)+\left(r^{-n} \mu(C(T, a, r, h) \sim A)\right)^{1 / n}\right)
\end{aligned}
$$

with a positive, finite number $\Gamma$ depending only on $m, n, Q, M$, and $\delta$, see Stein [18, p. 385]. Here $T_{n, 1}$ is the obvious generalisation of $T_{q}$ to Lorenz spaces.

A similar improvement is possible for part (1) using embeddings obtainable from [7, Lemma 7.14] and estimates for convolutions (cf. O'Neil [12]).

The proofs of the preceding theorem and of Lemma 3.15 (6) have been carefully chosen to facilitate the extension to Lorentz spaces. The only significant difference is the estimate of the auxiliary function $u$ occuring in the proof of Lemma 3.15 (6) which has to be replaced by $\|u\|_{L^{s^{*}, 1}\left(\mathcal{L}^{n}\right)} \leq \Gamma \mathcal{L}^{n}\left(\bigcup\left\{\bar{B}_{t_{i}}\left(\zeta_{i}\right): i \in I\right\}\right)^{1 / s}$ for $1 \leq s<n, s^{*}=s n /(n-s)$ and some positive, finite number $\Gamma$ depending only on $s$ and $n$. Assuming $I$ finite and $\left\{\bar{B}_{2 t_{i}}\left(x_{i}\right): i \in I\right\}$ to be disjointed, $u / 2$ is dominated by the Lipschitzian function with compact support mapping $x \in \mathbb{R}^{n}$ onto $\sum_{i \in I} \max \left\{0, t_{i}-\operatorname{dist}\left(x, \bar{B}_{t_{i}}\left(x_{i}\right)\right)\right\}$ to which the above mentioned embedding results can be applied to yield the estimate in question.

Corollary 4.8 Suppose $m, n, Q \in \mathbb{N}, 1 \leq M<\infty, 0<\delta \leq 1, a \in \mathbb{R}^{n+m}, 0<r<\infty, T \in$ $G(n+m, n), 1 \leq p \leq n, \mu$ is an integral $n$ varifold in an open superset of $C(T, a, 3 r, 3 r)$ satisfying $\left(H_{p}\right)$ and

$$
\begin{aligned}
& \psi=\|\delta \mu\| \text { if } p=1, \quad \psi=\left|\vec{H}_{\mu}\right|^{p} \mu \text { if } p>1, \\
& (Q-1+\delta) \omega_{n} r^{n} \leq \mu(C(T, a, r, r)) \leq(Q+1-\delta) \omega_{n} r^{n}, \\
& \mu(C(T, a, r,(1+\delta) r) \sim C(T, a, r,(1-\delta) r)) \leq(1-\delta) \omega_{n} r^{n}, \\
& \mu(C(T, a, 3 r, 3 r)) \leq M \omega_{n} r^{n} .
\end{aligned}
$$

Then the following two statements hold:

(1) If $p<n, 1 \leq q<n$, then

$$
\begin{aligned}
& H_{\frac{n q}{n-q}}(\mu, a, r, r, Q, T) \\
& \leq \Gamma_{(1)}\left(T_{q}(\mu, a, 3 r, 3 r, T)+\left(r^{p-n} \psi(C(T, a, 3 r, 3 r))\right)^{\frac{n-q}{q(n-p)}}\right)
\end{aligned}
$$

where $\Gamma_{(1)}$ is a positive, finite number depending only on $m, n, Q, M, \delta, p$, and $q$.

(2) If $p=n$ and $\psi(C(T, a, 3 r, 3 r)) \leq \varepsilon_{(2)}$ where $\varepsilon_{(2)}$ is a positive, finite number depending only on $m, n, Q, M$, and $\delta$, then

(a) $H_{\frac{n q}{n-q}}(\mu, a, r, r, Q, T) \leq \Gamma_{(2 \mathrm{a})} T_{q}(\mu, a, 3 r, 3 r, T)$ whenever $1 \leq q<n$, 
(b) $H_{\infty}(\mu, a, r, r, Q, T) \leq \Gamma_{(2 \mathrm{~b})} T_{q}(\mu, a, 3 r, 3 r, T)$ whenever $n<q \leq \infty$

where $\Gamma_{(2 \mathrm{a})}, \Gamma_{(2 \mathrm{~b})}$ are positive, finite numbers depending only on $m, n, Q, M, \delta$, and $q$.

Proof To prove part (1), assume $a=0, r=1$, define $q^{*}=n q /(n-q)$, and suppose that $\varepsilon=\varepsilon_{4.4}(m, n, Q, M, \delta)$. One only needs to consider the case that the right hand side is sufficiently small such that, using Hölder's inequality,

$$
\|\delta \mu\|(C(T, a, 3,3)) \leq \varepsilon r^{n-1}, \quad T_{1}(\mu, a, 3,3, T) \leq \varepsilon,
$$

since

$$
H_{q^{*}}(\mu, 0,1,1, Q, T) \leq \mu(C(T, 0,1,1))^{1 / q^{*}}+\omega_{n}^{1 / q} \leq M^{1 / q^{*}} \omega_{n}^{1 / q^{*}}+\omega_{n}^{1 / q} .
$$

The conclusion then follows from Theorem 4.4(1) in conjunction with Remarks 4.5 and 4.6.

Part (2) is proved similarly using Theorem 4.4(2).

Remark 4.9 In case $\mu$ additionally satisfies

$$
\mu\left(\left\{x \in C(T, a, r, r): \theta^{n}(\mu, x)=Q\right\}\right) \geq \delta \omega_{n} r^{n},
$$

there exists $z \in T^{\perp}$ such that for $P:=Q \mathcal{H}^{m}\left\llcorner\left\{x \in \mathbb{R}^{n+m}: T^{\perp}(x)=z\right\}\right.$

$$
H_{\frac{n q}{n-q}}(\mu, a, r, r, P) \leq \Gamma\left(T_{q}(\mu, a, 3 r, 3 r, T)+\left(r^{p-n} \psi(C(T, a, 3 r, 3 r))\right)^{\frac{n-q}{q(n-p)}}\right)
$$

provided $p<n, 1 \leq q<n$ where $\Gamma$ is a positive, finite number depending only on $m, n, Q$, $M, \delta, p$, and $q$.

In fact from Lemma 3.15 (2) (3) and the coarea formula [6, 3.2.22(3)] one obtains for the set $Y_{0}$ of all $y \in T \cap B_{r}(T(a))$ such that for some $x_{0} \in C(T, a, r, r)$ with $T\left(x_{0}\right)=y$

$$
\theta^{n}\left(\mu, x_{0}\right)=Q, \quad \theta^{n}(\mu, x)=0 \text { for } x \in T^{-1}(\{y\}) \cap C(T, a, r, r) \sim\left\{x_{0}\right\}
$$

the estimate

$$
\mathcal{L}^{1}\left(Y_{0}\right) \geq(2 \delta / 3) \omega_{n} r^{n}
$$

provided the right hand side of the inequality in question is suitably small (depending only on $m, n, Q, M, \delta, p$, and $q$ ), hence for any $Q$ valued plane $P^{\prime}$ parallel to $T$ such that

$$
\left(2 H_{\frac{n q}{n-q}}\left(\mu, a, r, r, P^{\prime}\right)\right)^{q} \leq(\delta / 3) \omega_{n}
$$

there holds

$$
\left((\delta / 3) \omega_{n}\right)^{1 / q-1 / n} \frac{\operatorname{diam} T^{\perp}\left(\operatorname{spt} P^{\prime}\right)}{2 r} \leq 2 H_{\frac{n q}{n-q}}\left(\mu, a, r, r, P^{\prime}\right)
$$

and suitable $z$ and $\Gamma$ are readily constructed.

A similar remark holds for the second part.

Example 4.10 Suppose $m=1, n=2, Q=1, a=0, \delta=1 / 4$,

$$
\begin{aligned}
& T=\left\{\left(x_{1}, x_{2}, x_{3}\right) \in \mathbb{R}^{3}: x_{3}=1 / 2\right\}, \\
& N=\left\{\left(x_{1}, x_{2}, x_{3}\right) \in \mathbb{R}^{3}: \cosh x_{3}=\left(x_{1}^{2}+x_{2}^{2}\right)^{1 / 2}\right\},
\end{aligned}
$$

$\mu=\mathcal{H}^{2}\llcorner(T \cup N)$ and $r$ slightly larger than 1. It is a classical fact that the catenoid $N$ is stationary, i.e. $\delta\left(\mathcal{H}^{2}\llcorner N)=0\right.$, hence $\delta \mu=0$. Therefore, considering the limit $r \downarrow 1$, one notes that $T_{q}(\mu, a, 3 r, 3 r, T)$ cannot be replaced by $T_{q}(\mu, a, r, r, T)$ in the conclusion of Corollary 4.8. It is not known to the author if such kind of behaviour can be excluded by introducing a smallness assumption on $T_{q}(\mu, a, 3 r, 3 r, T)$. 
Theorem 4.11 Suppose $m, n, Q \in \mathbb{N}, 0<\alpha \leq 1,1 \leq p \leq n$, $U$ is an open subset of $\mathbb{R}^{n+m}$, and $\mu$ is an integral $n$ varifold in $U$ satisfying $\left(H_{p}\right)$.

Then the following two statements hold:

(1) If $p<n, 1 \leq q_{1}<n, 1 \leq q_{2} \leq \min \left\{\frac{n q_{1}}{n-q_{1}}, \frac{1}{\alpha} \cdot \frac{n p}{n-p}\right\}$, then for $\mu$ almost all $a \in U$ with $\theta^{n}(\mu, a)=Q$ there holds

$$
\begin{aligned}
& \limsup _{r \downarrow 0} r^{-\alpha-1-n / q_{2}}\left\|\operatorname{dist}\left(\cdot-a, T_{a} \mu\right)\right\|_{L^{q_{2}}\left(\mu\left\llcorner B_{r}(a)\right)\right.} \\
& \quad \leq \Gamma_{(1)} \limsup _{r \downarrow 0} r^{-\alpha-n / q_{1}}\left\|T_{\mu}-T_{a} \mu\right\|_{L^{q_{1}}\left(\mu\left\llcorner B_{r}(a)\right)\right.}
\end{aligned}
$$

where $\Gamma_{(1)}$ is a positive, finite number depending only on $m, n, Q, q_{1}$, and $q_{2}$.

(2) If $p=n, n<q \leq \infty$, then for $\mu$ almost all $a \in U$ with $\theta^{n}(\mu, a)=Q$ there holds

$$
\begin{aligned}
& \underset{r \downarrow 0}{\limsup } r^{-\alpha-1}\left\|\operatorname{dist}\left(\cdot-a, T_{a} \mu\right)\right\|_{L^{\infty}\left(\mu\left\llcorner B_{r}(a)\right)\right.} \\
& \leq \Gamma_{(2)} \limsup _{r \downarrow 0} r^{-\alpha-n / q}\left\|T_{\mu}-T_{a} \mu\right\|_{L^{q}\left(\mu\left\llcorner B_{r}(a)\right)\right.}
\end{aligned}
$$

where $\Gamma_{(2)}$ is a positive, finite number depending only on $m, n, Q$, and $q$.

Proof For $a \in \mathbb{R}^{n+m}, 0<r<\infty$ such that $B_{7 r}(a) \subset U$ denote by $G_{r}(a)$ the set of all $x \in \bar{B}_{5 r}(a) \cap \operatorname{spt} \mu$ satisfying

$$
\|\delta \mu\|\left(\bar{B}_{\varrho}(x)\right) \leq\left(2 \gamma_{n}\right)^{-1} \mu\left(\bar{B}_{\varrho}(x)\right)^{1-1 / n} \text { whenever } 0<\varrho<2 r .
$$

To prove (1), one may assume first that $q_{2} \geq n /(n-1)$ possibly replacing $q_{2}$ by a larger number since $\min \left\{\frac{n q_{1}}{n-q_{1}}, \frac{1}{\alpha} \cdot \frac{n p}{n-p}\right\} \geq \frac{n}{n-1}$, and thus also that $q_{2}=n q_{1} /\left(n-q_{1}\right)$ possibly replacing $q_{1}$ by a smaller number. Define $M=6^{n} Q, \delta=1 / 2, q=q_{1}, q^{*}=q_{2}$,

$$
\varepsilon=\min \left\{\varepsilon_{4.4}(m, n, Q, M, \delta),\left(2 \gamma_{n}\right)^{-1}\right\}, \quad \Gamma_{1}=\Gamma_{4.4(1)}(m, n, Q, M, \delta, q) .
$$

Denote by $C_{i}$ for $i \in \mathbb{N}$ the set of all $x \in$ spt $\mu$ such that $B_{1 / i}(x) \subset U$ and

$$
\|\delta \mu\|\left(\bar{B}_{\varrho}(x)\right) \leq \varepsilon \mu\left(\bar{B}_{\varrho}(x)\right)^{1-1 / n} \text { whenever } 0<\varrho<1 / i .
$$

The conclusion will be shown for $a \in \operatorname{dmn} T_{\mu}$ such that

$$
\begin{gathered}
\theta^{n}(\mu, a)=Q, \quad \theta^{n-1}(\|\delta \mu\|, a)=0, \\
\lim _{r \downarrow 0} r^{-n^{2} /(n-p)} \mu\left(\bar{B}_{r}(x) \sim C_{i}\right)=0 \quad \text { for some } i \in \mathbb{N} .
\end{gathered}
$$

Note that according to $[6,2.9 .5]$ and $[11,2.9,2.10]$ with $s$ replaced by $n$ this is true for $\mu$ almost all $a \in U$ with $\theta^{n}(\mu, a)=Q$, fix such $a, i$, and abbreviate $T:=T_{a} \mu$.

For $a$ there holds

$$
\begin{gathered}
\lim _{r \downarrow 0} \frac{\mu(C(T, a, r, r))}{\omega_{n} r^{n}}=Q, \\
\lim _{r \downarrow 0} \frac{\mu(C(T, a, r, 3 r / 2) \sim C(T, a, r, r / 2))}{\omega_{n} r^{n}}=0
\end{gathered}
$$

and one can assume for some $0<\gamma<\infty$

$$
\underset{r \downarrow 0}{\limsup } r^{-\alpha} T_{q}(\mu, a, r, r, T)<\gamma .
$$


Noting $q \leq q^{*} \leq \frac{1}{\alpha} \cdot \frac{n p}{n-p}$, one chooses $0<s<\min \left\{(2 i)^{-1}, \operatorname{dist}\left(a, \mathbb{R}^{n+m} \sim U\right) / 7\right\}$ so small that for $0<\varrho<s$

$$
\begin{aligned}
& (Q-1 / 2) \omega_{n} \varrho^{n} \leq \mu(C(T, a, \varrho, \varrho)) \leq(Q+1 / 2) \omega_{n} \varrho^{n}, \\
& \mu(C(T, a, \varrho, 3 \varrho / 2) \sim C(T, a, \varrho, \varrho / 2)) \leq(1 / 2) \omega_{n} \varrho^{n}, \\
& \mu(C(T, a, 3 \varrho, 3 \varrho)) \leq \mu\left(\bar{B}_{5 \varrho}(a)\right) \leq \omega_{n} 6^{n} Q \varrho^{n}, \\
& \|\delta \mu\|(C(T, a, 3 \varrho, 3 \varrho)) \leq \varepsilon \varrho^{n-1}, \quad T_{1}(\mu, a, 3 \varrho, 3 \varrho, T) \leq \varepsilon, \\
& T_{q}(\mu, a, 3 \varrho, 3 \varrho, T)+\left(\varrho^{-n} \mu\left(C(T, a, \varrho, \varrho) \sim C_{i}\right)\right)^{1 / q} \leq 4 \gamma \varrho^{\alpha} ;
\end{aligned}
$$

in particular Theorem 4.4(1) can be applied to any such $\varrho$ with $r, h$ replaced by $\varrho, \varrho$. Also note that $G_{\varrho}(a) \cap C(T, a, \varrho, \varrho)$ equals the set $G$ defined in Theorem 4.4 with $r, h$ replaced by $\varrho, \varrho$ for $0<\varrho<s$. For each $0<\varrho<s$ use Remark 4.3 to choose a $Q$ valued plane $P_{\varrho}$ parallel to $T$ such that

$$
H_{q^{*}}\left(\mu\left\llcorner G_{\varrho}(a), a, \varrho, \varrho, P_{\varrho}\right) \leq 2 H_{q^{*}}\left(\mu\left\llcorner G_{\varrho}(a), a, \varrho, \varrho, Q, T\right),\right.\right.
$$

denote by $A_{\varrho}$ the $\mathcal{H}^{n}$ measurable set of all $x \in T \cap \bar{B}_{\varrho}(T(a))$ such that for some $R_{\varrho}(x), S_{\varrho}(x) \in Q_{Q}\left(\mathbb{R}^{n+m}\right)$

$$
\begin{aligned}
\left\|R_{\varrho}(x)\right\| & =\theta^{n}\left(P _ { \varrho } \llcorner C ( T , a , \varrho , \varrho ) , \cdot ) \mathcal { H } ^ { 0 } \left\llcornerT^{-1}(\{x\}),\right.\right. \\
\left\|S_{\varrho}(x)\right\| & =\theta^{n}\left(\mu \llcorner G _ { \varrho } ( a ) \cap C ( T , a , \varrho , \varrho ) , \cdot ) \mathcal { H } ^ { 0 } \left\llcornerT^{-1}(\{x\}),\right.\right.
\end{aligned}
$$

and by $g_{\varrho}: A_{\varrho} \rightarrow \mathbb{R}$ the $\mathcal{H}^{n}$ measurable functions defined by

$$
g_{\varrho}(x)=\mathcal{G}\left(R_{\varrho}(x), S_{\varrho}(x)\right) \text { for } x \in A_{\varrho} .
$$

By Remark 4.3 there exist $\mathcal{H}^{n}$ measurable subset $Y_{\varrho}$ of $A_{\varrho}$ such that

$$
\begin{aligned}
& 2 H_{q^{*}}\left(\mu\left\llcorner G_{\varrho}(a), a, \varrho, \varrho, P_{\varrho}\right) \geq \varrho^{-n / q}\left\|\operatorname{dist}\left(\cdot, \operatorname{spt} P_{\varrho}\right)\right\|_{L^{q^{*}}\left(\mu\left\llcorner G_{\varrho}(a) \cap C(T, a, \varrho, \varrho)\right)\right.}\right. \\
& +\varrho^{-n / q}\left\|g_{\varrho}\right\|_{L^{q^{*}}\left(\mathcal{H}^{n}\left\llcorner Y_{\varrho}\right)\right.}+\varrho^{-n / q} \mathcal{H}^{n}\left(T \cap \bar{B}_{\varrho}(T(a)) \sim Y_{\varrho}\right)^{1 / q} .
\end{aligned}
$$

Possibly replacing $s$ by a smaller number, one may assume for $0<\varrho<s$ that

$$
\left(2 H_{q^{*}}\left(\mu\left\llcorner G_{\varrho}(a), a, \varrho, \varrho, P_{\varrho}\right)\right)^{q} \leq 2^{-n-2} \omega_{n}\right.
$$

by Theorem 4.4(1) and also that

$$
\mu\left(C(T, a, \varrho, \varrho) \sim C_{i}\right) \leq 2^{-n-2} \omega_{n} \varrho^{n} .
$$

Noting $C_{i} \cap C(T, a, \varrho / 2, \varrho) \subset G_{\varrho}(a) \cap G_{\varrho / 2}(a)$, one obtains directly from the additional assumptions on $s$ that

$$
\begin{aligned}
& \mathcal{H}^{n}\left(T \cap \bar{B}_{\varrho}(T(a)) \sim Y_{\varrho}\right) \leq 2^{-n-2} \omega_{n} \varrho^{n}, \\
& \mathcal{H}^{n}\left(T \cap \bar{B}_{\varrho / 2}(T(a)) \sim Y_{\varrho / 2}\right) \leq 2^{-n-2} \omega_{n} \varrho^{n}, \\
& \mathcal{H}^{n}\left(\left\{x \in Y_{\varrho / 2} \cap Y_{\varrho}: S_{\varrho}(x) \neq S_{\varrho / 2}(x)\right\}\right) \\
& \quad \leq \mathcal{H}^{n}\left(T\left(\left\{x \in C(T, a, \varrho / 2, \varrho): \theta^{* n}(\mu, x) \geq 1\right\} \sim C_{i}\right)\right) \\
& \quad \leq \mu\left(C(T, a, \varrho, \varrho) \sim C_{i}\right) \leq 2^{-n-2} \omega_{n} \varrho^{n},
\end{aligned}
$$

hence for $B_{\varrho}:=Y_{\varrho} \cap Y_{\varrho / 2} \cap\left\{x: S_{\varrho}(x)=S_{\varrho / 2}(x)\right\}$

$$
\mathcal{H}^{n}\left(B_{\varrho}\right) \geq(1 / 4) \omega_{n}(\varrho / 2)^{n} \text { for } 0<\varrho<s,
$$


in particular

$$
\operatorname{dmn} R_{\varrho}=A_{\varrho} \supset Y_{\varrho} \supset B_{\varrho} \neq \emptyset, \quad \mathcal{G}\left(P_{\varrho}, Q \mathcal{H}^{n}\llcorner T) \leq Q^{1 / 2} \varrho .\right.
$$

By integration over the set $B_{\varrho}$ with respect to $\mathcal{H}^{n}$ one obtains

$$
\begin{aligned}
& \left((1 / 4) \omega_{n}(\varrho / 2)^{n}\right)^{1 / q-1 / n} \mathcal{G}\left(P_{\varrho}, P_{\varrho / 2}\right) \\
& \quad \leq\left\|g_{\varrho}\right\|_{L^{q^{*}}\left(\mathcal{H}^{n}\left\llcorner Y_{\varrho}\right)\right.}+\left\|g_{\varrho / 2}\right\|_{L^{q^{*}}\left(\mathcal{H}^{n}\left\llcorner Y_{\varrho / 2}\right)\right.} \\
& \quad \leq \varrho^{n / q} 4\left(H _ { q ^ { * } } \left(\mu\left\llcorner G_{\varrho}(a), a, \varrho, \varrho, Q, T\right)+H_{q^{*}}\left(\mu\left\llcorner G_{\varrho / 2}(a), \varrho / 2, \varrho / 2, Q, T\right)\right)\right.\right.
\end{aligned}
$$

for $0<\varrho<s$. Therefore Theorem 4.4(1) implies

$$
\mathcal{G}\left(P_{\varrho}, P_{\varrho / 2}\right) \leq \Gamma_{2} \gamma \varrho^{1+\alpha}
$$

where $\Gamma_{2}=2^{4+n / q+2 / q-2 / n} \omega_{n}^{1 / n-1 / q} \Gamma_{1}$, hence

$$
\mathcal{G}\left(Q \mathcal{H}^{n}\left\llcorner T, P_{\varrho}\right) \leq \sum_{i=0}^{\infty} \mathcal{G}\left(P_{2^{-i}} \varrho, P_{2^{-i-1}} \varrho\right) \leq 2 \Gamma_{2} \gamma \varrho^{1+\alpha}\right.
$$

because $\mathcal{G}\left(P_{\varrho}, Q \mathcal{H}^{n}\llcorner T) \rightarrow 0\right.$ as $\varrho \downarrow 0$. From the definition of the $q^{*}$ height of $\mu$ in $C(T, a, \varrho, \varrho)$ one obtains

$$
\begin{aligned}
& H_{q^{*}}\left(\mu \left\llcornerG_{\varrho}(a), a, \varrho, \varrho, Q \mathcal{H}^{n}\llcorner T)-H_{q^{*}}\left(\mu\left\llcorner G_{\varrho}(a), a, \varrho, \varrho, P_{\varrho}\right)\right.\right.\right. \\
& \quad \leq \varrho^{-n / q}\left(\mu(C(T, a, \varrho, \varrho))^{1 / q^{*}}+\mathcal{H}^{n}\left(Y_{\varrho}\right)^{1 / q^{*}}\right) \mathcal{G}\left(Q \mathcal{H}^{n}\left\llcorner T, P_{\varrho}\right) \leq \Gamma_{3} \gamma \varrho^{\alpha}\right.
\end{aligned}
$$

for $0<\varrho<s$ where $\Gamma_{3}=\omega_{n}^{1 / q^{*}} 2(Q+1)^{1 / q^{*}} 2 \Gamma_{2}$, hence

$$
\underset{\varrho \downarrow 0}{\limsup } \varrho^{-\alpha} H_{q^{*}}\left(\mu \left\llcornerG_{\varrho}(a), a, \varrho, \varrho, Q \mathcal{H}^{n}\llcorner T) \leq\left(8 \Gamma_{1}+\Gamma_{3}\right) \gamma\right.\right.
$$

by Theorem 4.4(1). Combining this with the fact that

$$
\lim _{\varrho \downarrow 0} \varrho^{-\alpha-1-n / q^{*}}\left\|\operatorname{dist}\left(\cdot-a, T_{a} \mu\right)\right\|_{L^{q^{*}}\left(\mu\left\llcorner B_{\varrho}(a) \sim G_{\varrho}(a)\right)\right.}=0,
$$

since $C_{i} \cap B_{\varrho}(a) \subset G_{\varrho}(a)$ and $\alpha q^{*}+n \leq n^{2} /(n-p)$, the conclusion follows.

(2) may be proved by a similar but simpler argument using Theorem 4.4(2) and [11, 2.5] instead of Theorem 4.4(1) and [11, 2.9, 2.10].

Remark 4.12 As in Remark 4.7, in (2) the $L^{q}$ norm can be replaced by $L^{n, 1}$, in particular $n=q=1$ is admissible. The latter fact can be derived without the use of Lorentz spaces, of course.

Remark 4.13 If $1 \leq p<n, 1 \leq q_{1} \leq q_{2}<\infty, \frac{1}{\alpha} \cdot \frac{n p}{n-p}<q_{2}$, then the conclusion of (1) fails for some $\mu$; in fact one can assume $q_{1}=q_{2}$ possibly enlarging $q_{1}$ and then take $\alpha_{2}=\alpha$ and $\alpha_{1}$ slightly larger than $\alpha_{2}$ in [11, 1.2]. Clearly, also in (2) the assumption $p=n$ cannot be weakened.

Acknowledgements The author acknowledges financial support via the Forschergruppe no. 469 of the Deutsche Forschungsgemeinschaft. The research was carried out while the author was a PhD student at the University of Tübingen and put in its final form while the author was at the AEI Golm and the ETH Zürich. AEI publication number: AEI-2008-064.

The author offers his thanks to Professor Reiner Schätzle for guiding him during the preparation of the underlying dissertation as well as interesting discussions about various mathematical topics. The author would also like to thank Professor Tom Ilmanen for his invitation to the ETH in Zürich in 2006, and for several interesting discussions concerning considerable parts of this work. 
Open Access This article is distributed under the terms of the Creative Commons Attribution Noncommercial License which permits any noncommercial use, distribution, and reproduction in any medium, provided the original author(s) and source are credited.

\section{References}

1. Allard, W.K.: On the first variation of a varifold. Ann. Math. (2) 95, 417-491 (1972)

2. Almgren, F.J., Jr.: Almgren's Big Regularity Paper, volume 1 of World Scientific Monograph Series in Mathematics. World Scientific Publishing Co. Inc., River Edge, NJ, 2000. $Q$-valued functions minimizing Dirichlet's integral and the regularity of area-minimizing rectifiable currents up to codimension 2, With a preface by Jean E. Taylor and Vladimir Scheffer

3. Anzellotti, G., Serapioni, R.P.: $\mathcal{C}^{k}$-rectifiable sets. J. Reine Angew. Math. 453, 1-20 (1994)

4. Brakke, K.A.: The Motion of a Surface by its Mean Curvature, volume 20 of Mathematical Notes. Princeton University Press, Princeton (1978)

5. De Lellis, C., Spadaro, E.N.: $Q$-valued functions revisited (2009). arXiv:0803.0060v2 [math.AP]

6. Federer, H.: Geometric Measure Theory. Die Grundlehren der mathematischen Wissenschaften, Band 153. Springer-Verlag New York Inc., New York (1969)

7. Gilbarg, D., Trudinger, N.S.: Elliptic Partial Differential Equations of Second Oorder. Classics in Mathematics. Springer-Verlag, Berlin, 2001. Reprint of the 1998 edition

8. Goblet, J.: A selection theory for multiple-valued functions in the sense of Almgren. Ann. Acad. Sci. Fenn. Math. 31(2), 297-314 (2006)

9. Lovász, L., Plummer, M.D.: Matching Theory, volume 121 of North-Holland Mathematics Studies. NorthHolland Publishing Co., Amsterdam, 1986. Annals of Discrete Mathematics, 29

10. Menne, U.: $\mathcal{C}^{2}$ rectifiability and $Q$ valued functions. PhD thesis, Universität Tübingen (2008). http:// tobias-lib.ub.uni-tuebingen.de/volltexte/2008/3518

11. Menne, U.: Some applications of the isoperimetric inequality for integral varifolds. Adv. Calc. Var. 2, 247269 (2009)

12. O’Neil, R.: Convolution operators and $L(p, q)$ spaces. Duke Math. J. 30, 129-142 (1963)

13. Schätzle, R.: Hypersurfaces with mean curvature given by an ambient Sobolev function. J. Differ. Geom. 58(3), 371-420 (2001)

14. Schätzle, R.: Quadratic tilt-excess decay and strong maximum principle for varifolds. Ann. Sci. Norm. Super. Pisa Cl. Sci. (5) 3(1), 171-231 (2004)

15. Schätzle, R.: Lower semicontinuity of the Willmore functional for currents. J. Differ. Geom. 81(2), 437456 (2009)

16. Simon, L.M.: Lectures on Geometric Measure Theory, volume 3 of Proceedings of the Centre for Mathematical Analysis, Australian National University. Australian National University Centre for Mathematical Analysis, Canberra, 1983

17. Stein, E.M.: Singular Integrals and Differentiability Properties of Functions. Princeton Mathematical Series, No. 30. Princeton University Press, Princeton (1970)

18. Stein, E.M.: Editor's note: the differentiability of functions in $\mathbf{R}^{n}$. Ann. Math. (2) 113(2), 383-385 (1981) 